\title{
Broadband Extended Array Response-Based Subspace Multiparameter Estimation Method for Multipolarized Wireless Channel Measurements
}

This paper was downloaded from TechRxiv (https://www.techrxiv.org).

\section{LICENSE}

CC BY 4.0

SUBMISSION DATE / POSTED DATE

$21-07-2020$ / 29-07-2020

\section{CITATION}

Yang, Bensheng; Zhang, Peize; Wang, Haiming; Wang, Cheng-Xiang; You, Xiaohu (2020): Broadband Extended Array Response-Based Subspace Multiparameter Estimation Method for Multipolarized Wireless Channel Measurements. TechRxiv. Preprint. https://doi.org/10.36227/techrxiv.12682061.v1

$\mathrm{DOI}$ 


\title{
Broadband Extended Array Response-Based Subspace Multiparameter Estimation Method for Multipolarized Wireless Channel
}

\section{Measurements}

\author{
Bensheng Yang, Student Member, IEEE, Peize Zhang, Student Member, IEEE, \\ Haiming Wang, Member, IEEE, Cheng-Xiang Wang, Fellow, IEEE, \\ and Xiaohu You, Fellow, IEEE
}

\begin{abstract}
The clustered delay line channel model, in which each cluster consists of a number of rays, is widely used for link-level evaluations in mobile communications. Multiple parameters of each ray, including delay, amplitude, cross polarization ratio (XPR), initial phases of four polarization combinations and the azimuth and elevation angles of arrival and departure, shall be known. These parameters are
\end{abstract}

This work was supported in part by the National Key R\&D Program of China under Grant 2020YFB1804901, the National Natural Science Foundation of China under Grants 61671145 and 61960206006, the Key R\&D Program of Jiangsu Province of China under Grant BE2018121, the High Level Innovation and Entrepreneurial Talent Introduction Program in Jiangsu, China, the Research Fund of National Mobile Communications Research Laboratory, Southeast University, under Grant 2020B01, and the Huawei Cooperation Project. (Corresponding author: Haiming Wang.)

B. S. Yang, P. Z. Zhang, and H. M. Wang are with the State Key Laboratory of Millimeter Waves, Southeast University, Nanjing 210096, China, and also Purple Mountain Laboratories, Nanjing 211111, China (e-mail: yangbensheng@seu.edu.cn, pzzhang@seu.edu.cn, hmwang@seu.edu.cn).

C. X. Wang and X. H. You are with the National Mobile Communications Research Laboratory, Southeast University, Nanjing 210096, China, and also with Purple Mountain Laboratories, Nanjing 211111, China (e-mail: chxwang@seu.edu.cn, xhyu@seu.edu.cn).

Color versions of one or more of the figures in this paper are available online at http://ieeexplore.ieee.org.

Digital Object Identifier 
measured using a channel sounder. The number of rays in every cluster is usually greater than the number of elements in the antenna array of the channel sounder, which represents a challenging issue in multipolarized channel measurements. A new subspace estimation method based on the broadband extended array response of an electromagnetic vector antenna array is proposed to resolve a large number of rays. The interelement spacing of the array can be greater than half the carrier wavelength, which reduces interelement coupling and simplifies the array design, especially for millimeter wave bands. First, the delay of each cluster is estimated using the reference antenna element. Then, the 2D angles of every ray are estimated using the classic rank-deficient multiple signal classification (MUSIC). Lastly, the initial phases, XPR and amplitude of every ray is estimated. Simulation results validate the proposed method.

\section{Index Terms}

Broadband extended array response (BEAR), channel measurements, cross polarization ratio, electromagnetic vector antenna array (EMVAA), subspace estimation method.

\section{INTRODUCTION}

The performance of wireless transmission in mobile communication systems is directly dependent on the channel propagation characteristics. Therefore, a multiparameter estimation method for in-depth analysis of channel propagation characteristics and channel modeling of the mobile communication systems is important [1], [2]. In 2016, the third generation partnership project (3GPP) started a study item on channel models [3], [4]. In this study item, delay, two dimensional (2D) ${ }^{1}$ angles of departure (AoD), 2D angles of arrival (AoA) and cross polarization ratio (XPR) are essential small-scale parameters [5]. An accurate and efficient multiparameter estimation method is of great significance in channel measurements. In addition, polarization must be considered in channel modeling. Antenna polarization modeling is also advocated by 3 GPP.

Previous works have proved that multipolarized multiple input multiple output (MIMO) outperforms the corresponding unipolarized MIMO in terms of channel capacity in many situations [6]-[8]. Moreover, dual-polarized antenna configurations outperform single polarization in terms of spatial correlation [9]. Due to these advantages, the multipolarized MIMO is considered to be a promising technique for next-generation mobile communication systems. There has been

\footnotetext{
${ }^{1}$ Two dimensions mean the azimuth and elevation.
} 
increasing interest in polarized channel modeling and measurement, especially for multipolarized MIMO systems [10]-[13].

Multiparameter estimation methods, such as maximum likelihood (ML) algorithm [14], multiple signal classification (MUSIC) [15], estimation of signal parameters via rotational invariance techniques (ESPRIT) and space-alternating generalized expectation-maximization (SAGE) [16], [17], have been investigated intensively in unipolarized array signal processing. In [16], [18], [19], the delay and AoA are estimated via a subspace-based method, the number of total rays can be greater than the number of antenna elements but not the number of rays in one cluster ${ }^{2}$. In [17], [20], the delay, AoA, and fading coefficient are estimated using the SAGE algorithm. The SAGE and MUSIC algorithms are compared in [20] using measurement data. The multipolarized array signal processing algorithm has also received substantial attention, typically with respect to the electromagnetic vector antenna (EMVA) [21]-[25].

An EMVA is a hexapolarized antenna; however, due to the particularity of the EMVA structure, a collocated EMVA (C-EMVA) is complicated to construct [26]. The major difficulties originate from the antenna feeding method and the elimination of mutual coupling. To address these problems, a C-EMVA is usually reconstructed as a distributed EMVA (D-EMVA) or simplified to a dual- or triple-polarized antenna. The D-EMVA not only simplifies the antenna design but also suppresses the mutual coupling between the antenna elements. In a D-EMVA, the antenna elements are spatially distributed with a known distance [27].

However, a major difficulty in channel measurements is the parameter estimation of rays using multipolarized arrays when the number of rays in the cluster is greater than the number of array elements. The number of rays in one cluster often exceeds the number of antenna elements in the channel sounder. The existing multiparameter estimation methods usually do not work under such conditions or suffer from low robustness. Although this problem can be addressed by virtual MIMO indirectly, which is implied by shifting the antenna in the space mechanically, virtual MIMO is inconvenient and leads to long measurement time. Therefore, an efficient method to overcome this difficulty with lower hardware complexity is needed.

In this study, a broadband extended array response (BEAR)-based subspace method is proposed

\footnotetext{
${ }^{2} \mathrm{~A}$ cluster is defined as the path that can be resolved in the temporal domain. The number of rays in one path can be larger than the number of antenna array elements.
} 
to address the difficulty of using the EMVA array (EMVAA). The dimension of the steering vector can be increased substantially via BEAR. Traditionally, the AoA is obtained in the space domain, which requires the array element spacing to be smaller than half the carrier wavelength. This requirement increases both the complexity of the channel sounding system and the mutual coupling between the antenna elements. The limitation of interelement spacing can be overcome by the proposed method. The antenna array shape and interelement spacing are more flexible than in the traditional method. Furthermore, these advantages are more apparent in millimeter wave MIMO channel measurement systems. The main contributions of this work are summarized as follows.

- The BEAR-based subspace estimation method is proposed to estimate the delay, 2D AoD, 2D AoA, initial phases of the four polarization combination state, amplitude and XPR in multipath environments using a multipolarized antenna array (EMVAA). Specially, the XPR of each ray is estimated in detail, rather than an approximate result with the power ratio of each polarized antenna element. Most of the parameters related to channel coefficient generation in the $3 \mathrm{GPP}$ protocol can be estimated.

- By means of the proposed method, the radio frequency (RF) module of the channel sounding system is simplified and the mutual coupling in the antenna array is suppressed because the interelement spacing can be larger than half the carrier wavelength. Moreover, 2D angles can be estimated via linear EMVAA with low hardware complexity. Considering the complexity of the broadband loops in antenna design, the proposed method still works well with only dual- or triple- polarized antenna arrays.

- The number of rays in one cluster is greater than the number of antenna elements, which is a common difficulty in channel parameter estimation.The proposed BEAR-based method utilizes the response in the space-frequency-polarization (SFP) domain to solve this problem. The maximum number of rays that the proposed method can resolve is also given.

Notations: Upper (Lower) bold-face letters are used to denote matrices (vectors). A $K \times K$ identity matrix is denoted as $\mathbf{I}_{K} \cdot \mathbf{1}_{M \times N}$ represents the $M \times N$ matrix with all elements equal to 1. The $k$ th column vector of an identity matrix is a unit vector denoted as $\mathbf{e}_{k}$. Superscript $(\cdot)^{\mathrm{H}}$ denotes the Hermitian transpose, and $(\cdot)^{\mathrm{T}}$ denotes transpose. The Hadamard product, Khatri-Rao product, and Kronecker product are, respectively, denoted by $\odot, \oplus$, and $\otimes$. A diagonal matrix 


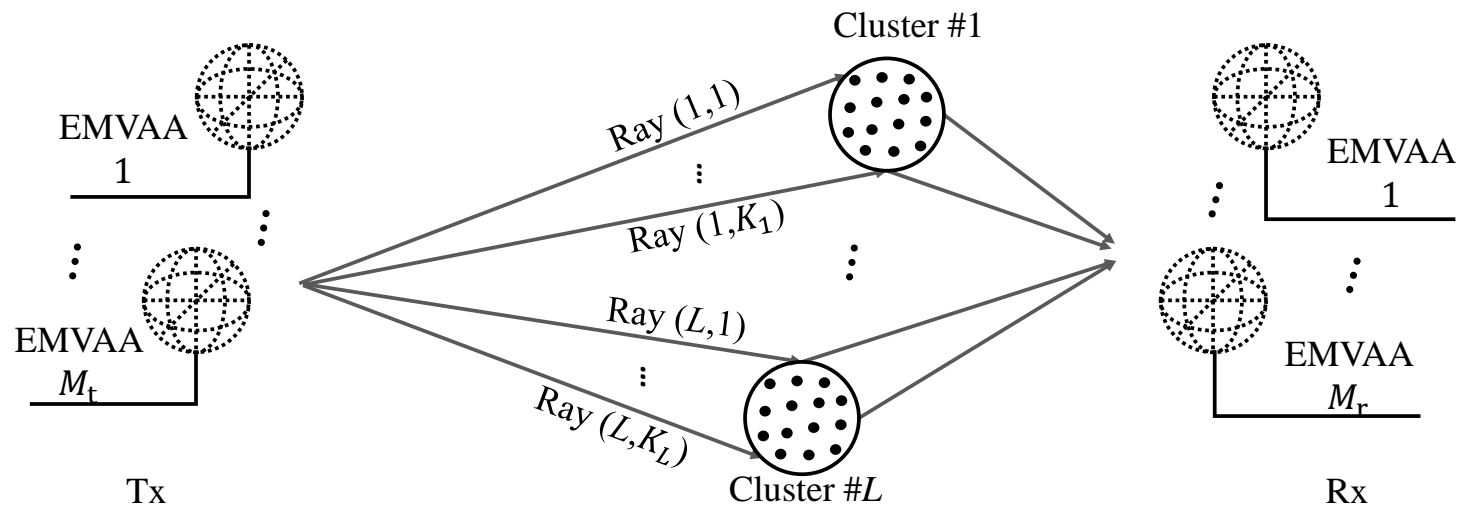

Fig. 1. Diagram of a CDL channel model.

with all entries of $\mathbf{x}$ on the diagonal is denoted by $\operatorname{diag}\{\mathbf{x}\}$. The phase angle of a complex number $x$ is denoted by $\arg \{x\}$. The pseudoinverse of $\mathbf{X}$ is represented as $\mathbf{X}^{\dagger}$.

\section{System Model}

In this section, the clustered delay line (CDL) channel model, multipolarized antenna system and the received signal model are presented. Subsequently, the SFP domain channel response of the wireless channel measurement system is obtained.

\section{A. Channel Model}

The CDL model is defined for the full frequency range from $0.5 \mathrm{GHz}$ to $100 \mathrm{GHz}$, with a maximum bandwidth of $2 \mathrm{GHz}$, which is used mainly for link-level simulations [3]. In this channel model, the parameters that need to be estimated are the delays of clusters, 2D AoD, 2D AoA, initial phases of the four polarization combination state, amplitude and XPR of rays. These parameters are defined in this subsection. A total of $K$ rays in $L$ clusters are considered for the multipath environment, where $K=\sum_{l=1}^{L} K_{l}$, with $K_{l}$ being the number of rays in the lth cluster. As shown in Fig. 1, the rays in the lth cluster arrive at the receiver with different AoDs and AoAs but approximately the same time delay $\tau_{l}$. Denote the $k$ th ray in the $l$ th cluster as the $k_{l}$ th ray. The azimuth AoA and AoD (AAoA and AAoD) of the $k_{l}$ th ray are denoted by $\varphi_{\mathrm{r}, l k}$ and $\varphi_{\mathrm{t}, l k}$. The elevation AoA and AoD (EAoA and EAoD) of the $k_{l}$ th ray are denoted by $\theta_{\mathrm{r}, l k}$ and $\theta_{\mathrm{t}, l k}$. The angle definition is given in Fig. 2 (a). Define $\Theta_{\mathrm{t}, l k} \triangleq\left\{\varphi_{\mathrm{t}, l k}, \theta_{\mathrm{t}, l k}\right\}$ and 
$\Theta_{\mathrm{r}, l k} \triangleq\left\{\varphi_{\mathrm{r}, l k}, \theta_{\mathrm{r}, l k}\right\}$. The direction cosine of AoD is denoted by

$$
\mathbf{u}_{\mathrm{t}, l k}\left(\Theta_{\mathrm{t}, l k}\right)=\left[\sin \theta_{\mathrm{t}, l k} \cos \varphi_{\mathrm{t}, l k}, \sin \theta_{\mathrm{t}, l k} \sin \varphi_{\mathrm{t}, l k}, \cos \theta_{\mathrm{t}, l k}\right]^{\mathrm{T}} .
$$

For the AoA, the direction cosine is $\mathbf{u}_{\mathrm{r}, l k}\left(\Theta_{\mathrm{r}, l k}\right)$, which is obtained by substituting the subscript ' $t$ ' in (1) with ' $r$ '. The ray may change polarization status during propagation. Such process is represented by

$$
\mathbf{T}\left(\Xi_{l k}\right)=\left[\begin{array}{cc}
e^{j \omega_{l k}^{\mathrm{vv}}} & \sqrt{\kappa_{l k}^{-1}} e^{j \omega_{l k}^{\mathrm{vh}}} \\
\sqrt{\kappa_{l k}^{-1}} e^{j \omega_{l k}^{\mathrm{hv}}} & e^{j \omega_{l k}^{\mathrm{hh}}}
\end{array}\right]
$$

where $\Xi_{l k} \triangleq\left\{\kappa_{l k}, \boldsymbol{\omega}_{l k}^{\mathrm{hh}}, \boldsymbol{\omega}_{l k}^{\mathrm{hv}}, \boldsymbol{\omega}_{l k}^{\mathrm{vh}}, \boldsymbol{\omega}_{l k}^{\mathrm{vv}}\right\}$ with $\kappa_{l k}$ representing the XPR of the $k_{l}$ th ray, $\left\{\boldsymbol{\omega}_{l k}^{\mathrm{hh}}, \boldsymbol{\omega}_{l k}^{\mathrm{hv}}, \boldsymbol{\omega}_{l k}^{\mathrm{vh}}, \boldsymbol{\omega}_{l k}^{\mathrm{vv}}\right\}$ being the different initial phases of the horizontal and vertical polarization combinations for the $k_{l}$ th ray.

\section{B. Antenna Pattern and Array Configuration}

In practical channel measurements, the gains of the transmit and receive antenna elements usually vary with the departure and incident angle. The antenna gains are taken into consideration in the received signal modeling progress to de-embed the influence of the antenna pattern. For a D-EMVA array (D-EMVAA), the dipoles and loops are arranged in different directions, so each element of a D-EMVA has a unique antenna gain. Here, the gains of the transmitting D-EMVA from $\Theta_{\mathrm{t}}$ are denoted as $G_{\mathrm{t}, \mathrm{ex}}\left(\Theta_{\mathrm{t}}\right), G_{\mathrm{t}, \mathrm{ey}}\left(\Theta_{\mathrm{t}}\right), G_{\mathrm{t}, \mathrm{ez}}\left(\Theta_{\mathrm{t}}\right), G_{\mathrm{t}, \mathrm{hx}}\left(\Theta_{\mathrm{t}}\right), G_{\mathrm{t}, \mathrm{hy}}\left(\Theta_{\mathrm{t}}\right)$ and $G_{\mathrm{t}, \mathrm{hz}}\left(\Theta_{\mathrm{t}}\right)$. Similarly for the AoA, the gains are denoted by substituting the subscript ' $t$ ' with 'r'.

In this work, a multipolarized MIMO system with $M_{\mathrm{t}}$ and $M_{\mathrm{r}}$ D-EMVAs on the transmitting side and receiving side is considered. Fig. 2(a) shows the structure of the applied antenna arrays where the dashed lines depict the C-EMVA. The definition of the azimuth and elevation angles is shown in Fig. 2(b). The coordinate of the $m$ th C-EMVA can be denoted as $\mathbf{r}_{m}=\left[r_{m, \mathrm{x}}, r_{m, \mathrm{y}}, r_{m, \mathrm{z}}\right]$. In addition, the reference coordinates of the six elements are given as $\mathbf{r}_{\mathrm{ex}}, \mathbf{r}_{\mathrm{ey}}, \mathbf{r}_{\mathrm{ez}}, \mathbf{r}_{\mathrm{hx}}, \mathbf{r}_{\mathrm{hy}}$, and $\mathbf{r}_{\mathrm{hz}}$ for a D-EMVA. In this study, the interelement spacing of the array can be larger than a half wavelength at the frequency of operation. Thus, the mutual coupling effect is effectively reduced, and the complicated array design is simplified. 


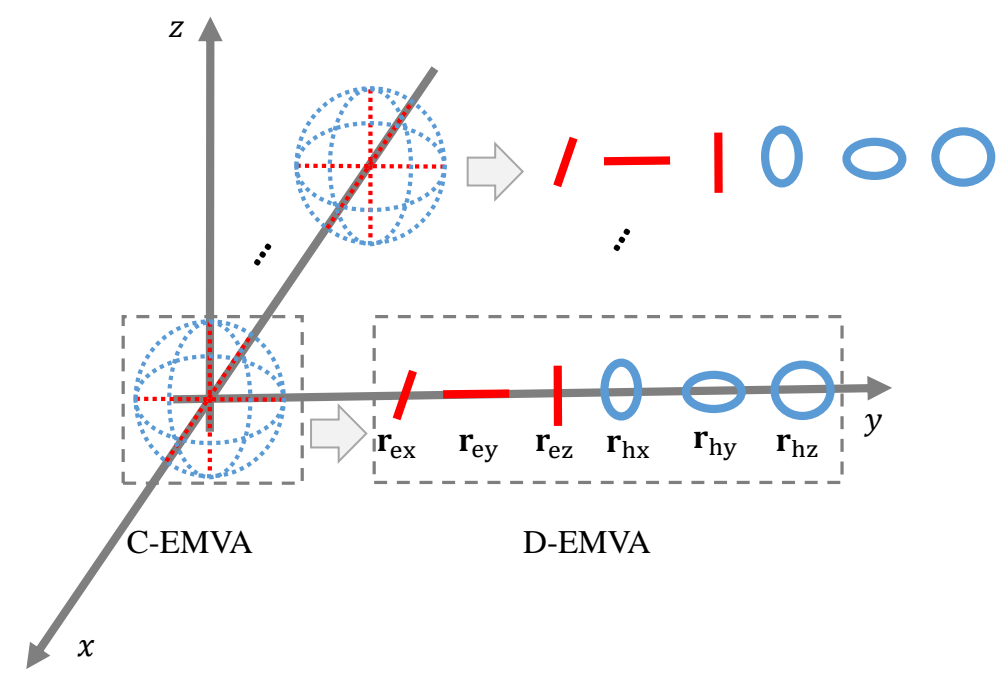

(a)

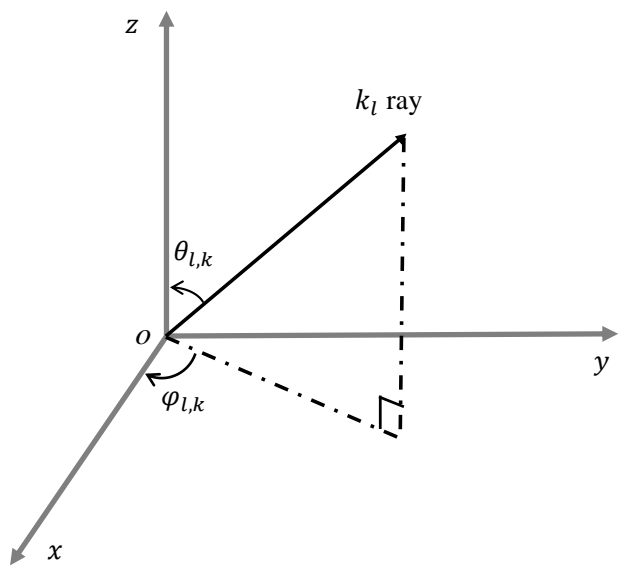

(b)

Fig. 2. EMVA configuration and angle definition. (a) Configuration of the C-EMVA and D-EMVA; (b) Definition of the azimuth and elevation angles.

\section{Transmit-Receive Signal Model}

As shown in Fig. 3, the transmitting sequence of the $\left(m_{\mathrm{t}}, i\right)$ th antenna ${ }^{3}$ in the $l_{\text {seq }}$ th subsequence can be expressed as a row vector $\mathbf{s}_{m_{\mathrm{t}}, i}\left[\left(l_{\mathrm{seq}}-1\right) N_{\mathrm{s}}+n_{\mathrm{s}} T\right], n_{\mathrm{s}}=1, \ldots, N_{\mathrm{s}}$ and $l_{\mathrm{seq}}=$ $1, \ldots, L_{\text {seq }}$, which is denoted as $s_{m_{\mathrm{t}}, i, l_{\mathrm{seq}}}\left(n_{\mathrm{s}} T\right)$ compactly. $T$ is the code width, and $N_{\mathrm{s}}$ is the length of the subsequence, which is also equal to the number of discrete Fourier transform (DFT) points. In practical implementations, the DFT uses fast Fourier transform (FFT). Note

${ }^{3}$ The $\left(m_{\mathrm{t}}, i\right)$ th antenna is defined as the $i$ th element of the $m_{\mathrm{t}}$ th D-EMVA, where $m_{\mathrm{t}}=1, \ldots, M_{\mathrm{t}}$ and $i=1, \ldots, 6$. 


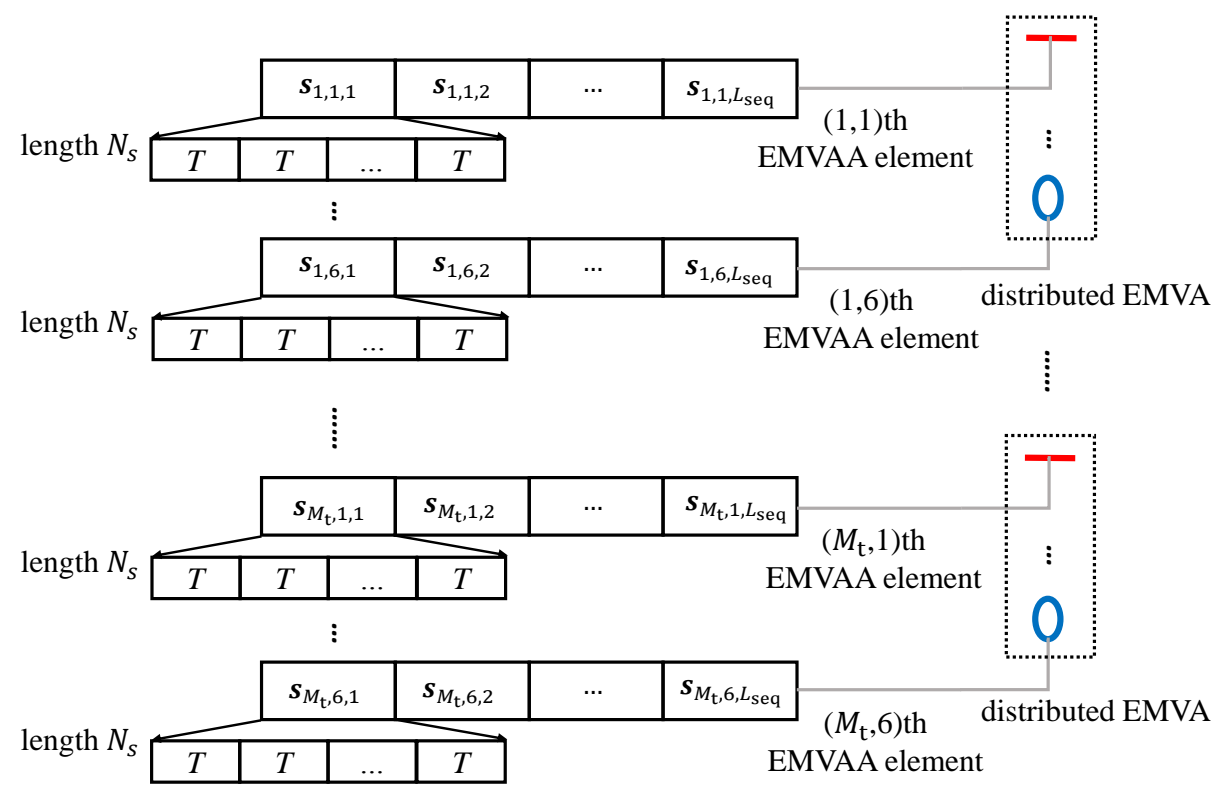

Fig. 3. Transmitting sequence structure of a snapshot.

that $L_{\text {seq }} \geq 6 M_{\mathrm{t}}$. The transmitting sequences for all transmitting antennas in matrix form can be denoted as

$$
\mathbf{S}=\left[\mathbf{S}_{1}, \mathbf{S}_{2}, \ldots, \mathbf{S}_{L_{\text {seq }}}\right]
$$

where

$$
\mathbf{S}_{l_{\mathrm{seq}}}=\left[\mathbf{s}_{l_{\mathrm{seq}} 1}, \ldots, \mathbf{s}_{l_{\mathrm{seq}} N_{\mathrm{s}}}\right]
$$

with $\mathbf{s}_{l_{\text {seq }} n_{\mathrm{s}}}$ being the $n_{\mathrm{s}}$ th code of the $l_{\text {seq }}$ th subsequence for all transmitting antennas, which is defined as

$$
\mathbf{s}_{l_{\mathrm{seq}} n_{\mathrm{s}}}=\left[s_{11 l_{\mathrm{seq}}}\left(n_{\mathrm{s}} T\right), \ldots, s_{M_{\mathrm{t}} 6 l_{\mathrm{seq}}}\left(n_{\mathrm{s}} T\right)\right]^{\mathrm{T}}
$$

Thus, the $n_{\mathrm{s}}$ sample of the $l_{\text {seq }}$ subsequence in the $n$th snapshot of the received signal can be denoted by (6), where $\mathbf{z}_{n}$ is the complex white Gaussian noise of the $n$th snapshot and $\alpha_{n, l k}$ represents the fading coefficient of the $k_{l}$ th ray in the $n$th snapshot. The channel is considered as time-varying for different snapshots.

$$
\begin{aligned}
\mathbf{y}_{n n_{\mathrm{s}} l_{\mathrm{seq}}}\left(f, \boldsymbol{\Theta}_{\mathrm{r}}, \boldsymbol{\Theta}_{\mathrm{t}}, \boldsymbol{\Xi}\right)=\sum_{l=1}^{L} \sum_{k=1}^{K_{l}} \alpha_{n, l k} \mathbf{a}_{\mathrm{r}}\left(f, \Theta_{\mathrm{r}, l k}\right) \otimes\left[\mathbf{g}_{\mathrm{r}}^{\mathrm{T}}\left(\Theta_{\mathrm{r}, l k}\right) \oplus \mathbf{A}_{\mathrm{pdr}}^{\mathrm{T}}\left(f, \Theta_{\mathrm{r}, l k}\right)\right]^{\mathrm{T}} \mathbf{T}\left(\Xi_{l k}\right) \\
\cdot \mathbf{a}_{\mathrm{t}}^{\mathrm{T}}\left(f, \Theta_{\mathrm{t}, l k}\right) \otimes\left[\mathbf{g}_{\mathrm{t}}^{\mathrm{T}}\left(\Theta_{\mathrm{t}, l k}\right) \oplus \mathbf{A}_{\mathrm{pdt}}^{\mathrm{T}}\left(f, \Theta_{\mathrm{t}, l k}\right)\right] \mathbf{s}_{l_{\mathrm{seq}}, n_{\mathrm{s}}}\left(n_{\mathrm{s}} T-\tau_{l}\right)+\mathbf{z}_{n}
\end{aligned}
$$


Let $\mathbf{a}_{\mathrm{t}}\left(f, \Theta_{\mathrm{t}, l k}\right)$ be the space-frequency domain steering vector of the $k_{l}$ th ray for the AoD. It can be expressed as

$$
\mathbf{a}_{\mathbf{t}}\left(f, \Theta_{\mathrm{t}, l k}\right)=\left[e^{-j 2 \pi f \frac{\mathbf{r}_{1} \mathbf{u}_{\mathrm{t}, l k}}{c}}, \ldots, e^{-j 2 \pi f \frac{\mathbf{r}_{M_{\mathrm{t}}} \mathbf{u}_{\mathrm{t}, l k}}{c}}\right]^{\mathrm{T}}
$$

where $c$ is the speed of light. For the AoA, it is denoted by $\mathbf{a}_{\mathbf{r}}\left(f, \Theta_{\mathrm{r}, l k}\right) \cdot \mathbf{g}_{\mathrm{t}}\left(\Theta_{\mathrm{t}, l k}\right)$ is the antenna gains vector of a D-EMVA for the AoD, which is written as

$\mathbf{g}_{\mathrm{t}}\left(\Theta_{\mathrm{t}, l k}\right)=\left[G_{\mathrm{t}, \mathrm{ex}}\left(\Theta_{\mathrm{t}, l k}\right), G_{\mathrm{t}, \mathrm{ey}}\left(\Theta_{\mathrm{t}, l k}\right), G_{\mathrm{t}, \mathrm{ez}}\left(\Theta_{\mathrm{t}, l k}\right), G_{\mathrm{t}, \mathrm{hx}}\left(\Theta_{\mathrm{t}, l k}\right), G_{\mathrm{t}, \mathrm{hy}}\left(\Theta_{\mathrm{t}, l k}\right), G_{\mathrm{t}, \mathrm{hz}}\left(\Theta_{\mathrm{t}, l k}\right)\right]^{\mathrm{T}}$.

For the AoA, $\mathbf{g}_{\mathrm{t}}\left(\Theta_{\mathrm{t}, l k}\right)$ is replaced by $\mathbf{g}_{\mathrm{r}}\left(\Theta_{\mathrm{r}, l k}\right)$. Let $\mathbf{A}_{\mathrm{pdt}}\left(f, \Theta_{\mathrm{t}, l k}\right)$ be the SFP domain steering matrix of the $k_{l}$ th ray for a D-EMVA in the transmitter. It can be expressed as

$$
\mathbf{A}_{\mathrm{pdt}}\left(f, \Theta_{\mathrm{t}, l k}\right)=\mathbf{D}_{\mathrm{t}}\left(f, \Theta_{\mathrm{t}, l k}\right) \boldsymbol{\Omega}_{\mathrm{t}}\left(\Theta_{\mathrm{t}, l k}\right)
$$

where $\mathbf{D}_{\mathrm{t}}\left(f, \Theta_{\mathrm{t}, l k}\right)$ is the diagonal phase shift matrix of a D-EMVA for the AoD, i.e.,

$$
\mathbf{D}_{\mathrm{t}}\left(f, \Theta_{\mathrm{t}, l k}\right)=\operatorname{diag}\left\{\mathbf{d}_{\mathrm{t}, l k}\left(f, \Theta_{\mathrm{t}, l k}\right)\right\}
$$

The phase shift vector $\mathbf{d}_{\mathrm{t}, l k}\left(f, \Theta_{\mathrm{t}, l k}\right)$ is denoted as (11).

$\mathbf{d}_{\mathrm{t}, l k}\left(f, \Theta_{\mathrm{t}, l k}\right)=\left[e^{-j 2 \pi f \frac{\mathbf{r}_{\mathbf{e x}} \mathbf{u}_{\mathrm{t}, l k}}{c}}, e^{-j 2 \pi f \frac{\mathbf{r}_{\mathbf{r y y}} \mathbf{u}_{\mathrm{t}, l k}}{c}}, e^{-j 2 \pi f \frac{\mathbf{r}_{\mathbf{e z}} \mathbf{u}_{\mathrm{t}, l k}}{c}}, e^{-j 2 \pi f \frac{\mathbf{r}_{\mathbf{h x}} \mathbf{u}_{\mathrm{t}, l k}}{c}}, e^{-j 2 \pi f \frac{\mathbf{r}_{\mathbf{h y}} \mathbf{u}_{\mathrm{t}, l k}}{c}}, e^{-j 2 \pi f \frac{\mathbf{r}_{\mathrm{hz}} \mathbf{u}_{\mathrm{t}, l k}}{c}}\right]^{\mathrm{T}}$

$\Omega_{\mathrm{t}}$ is the space-polarization domain steering matrix for the C-EMVA, which is expressed as

$$
\Omega\left(\Theta_{\mathrm{t}, l k}\right)=\left[\begin{array}{cc}
\cos \varphi_{\mathrm{t}, l k} \cos \theta_{\mathrm{t}, l k} & -\sin \varphi_{\mathrm{t}, l k} \\
\sin \varphi_{\mathrm{t}, l k} \cos \theta_{\mathrm{t}, l k} & \cos \varphi_{\mathrm{t}, l k} \\
-\sin \theta_{\mathrm{t}, l k} & 0 \\
-\sin \varphi_{\mathrm{t}, l k} & -\cos \varphi_{\mathrm{t}, l k} \cos \theta_{\mathrm{t}, l k} \\
\cos \varphi_{\mathrm{t}, l k} & -\sin \varphi_{\mathrm{t}, l k} \cos \theta_{\mathrm{t}, l k} \\
0 & \sin \theta_{\mathrm{t}, l k}
\end{array}\right]
$$

For the AoA, the corresponding expressions are denoted by $\mathbf{A}_{\mathrm{pdr}}\left(f, \Theta_{\mathrm{r}, l k}\right), \Omega_{\mathrm{r}}\left(\Theta_{\mathrm{r}, l k}\right), \mathbf{D}_{\mathrm{r}}\left(f, \Theta_{\mathrm{r}, l k}\right)$ and $\mathbf{d}_{\mathrm{r}, l k}\left(f, \Theta_{\mathrm{r}, l k}\right)$. 


\section{Channel Response in the SFP Domain}

The received signal $\mathbf{Y}_{n l_{\text {seq }}}$ in the $n$th snapshot consisting of $L_{\text {seq }}$ different subsequences, i.e.,

$$
\mathbf{Y}_{n l_{\text {seq }}}=\left[\mathbf{y}_{n 1 l_{\text {seq }}}, \mathbf{y}_{n 2 l_{\text {seq }}}, \ldots, \mathbf{y}_{n N_{\mathrm{s}} l_{\text {seq }}}\right]
$$

where $\mathbf{Y}_{n l_{\text {seq }}}\left(f, \boldsymbol{\Theta}_{\mathrm{t}}, \boldsymbol{\Theta}_{\mathrm{r}}, \boldsymbol{\Xi}\right)$ and $\mathbf{y}_{n n_{\mathrm{s}} l_{\mathrm{seq}}}\left(f, \boldsymbol{\Theta}_{\mathrm{t}}, \boldsymbol{\Theta}_{\mathrm{r}}, \boldsymbol{\Xi}\right)$ are replaced by $\mathbf{Y}_{n l_{\mathrm{seq}}}$ and $\mathbf{y}_{n n_{\mathrm{s}} l_{\mathrm{seq}}}$ for simplicity, respectively. Performing the DFT on each row of $\mathbf{Y}_{n l_{\text {seq }}} \in C^{6 M_{\mathrm{r}} \times N_{\mathrm{s}}}$ yields

$$
\mathbf{Y}_{n l_{\mathrm{seq}} f}=\left[\mathbf{y}_{n l_{\mathrm{seq}} f_{1}}, \mathbf{y}_{n l_{\mathrm{seq}} f_{2}}, \ldots, \mathbf{y}_{n l_{\mathrm{seq}} f_{N_{\mathrm{s}}}}\right]
$$

Then, the received signal of the $n$th snapshot in frequency bin $f_{i}$ can be obtained as

$$
\begin{aligned}
\mathbf{Y}_{n f_{i}} & =\left[\mathbf{y}_{n 1 f_{i}}, \mathbf{y}_{n 2 f_{i}}, \ldots, \mathbf{y}_{n L_{\mathrm{seq}} f_{i}}\right] \\
& =\mathbf{H}_{n f_{i}} \tilde{\mathbf{S}}_{f_{i}}+\mathbf{Z}_{n f_{i}}
\end{aligned}
$$

where $\mathbf{Y}_{n f_{i}} \in \mathbb{C}^{6 M_{\mathrm{r}} \times L_{\mathrm{seq}}}, \mathbf{H}_{n f_{i}}$ and $\mathbf{Z}_{n f_{i}}$ represent the channel response and additive white Gaussian noise in the $n$th snapshot in frequency bin $f_{i}$. The standard deviation of the noise is $\sigma_{\mathrm{n}}$ with mean zero. $\tilde{\mathbf{S}}_{f_{i}}$ is the transmitting signal in frequency bin $f_{i}$ for all subsequences, which can be expressed as

$$
\tilde{\mathbf{S}}_{f_{i}}=\left[\mathcal{F} \mathrm{S}_{1}, \mathcal{F} \mathrm{S}_{2}, \ldots, \mathcal{F} \mathbf{S}_{L_{\mathrm{seq}}}\right] \mathbf{J}_{f_{i}}
$$

where $\mathcal{F}$ stands for the DFT matrix and $\mathbf{J}_{f_{i}}$ is the selection matrix that selects the signal of the frequency bin $f_{i}$, which is defined as $\mathbf{J}_{f_{i}}=\mathbf{I}_{L_{\mathrm{seq}}} \otimes \mathbf{e}_{i}$ with $\mathbf{e}_{i}$ being the $i$ th column of $\mathbf{I}_{N_{\mathrm{s}}}$. Each row of $\tilde{\mathbf{S}}_{f_{i}}$ is orthogonal to each other, which satisfies

$$
\mathbf{I}_{M_{t}}=\tilde{\mathbf{S}}_{f_{i}} \tilde{\mathbf{S}}_{f_{i}}^{\mathrm{H}}
$$

Let $\mathbf{h}_{n f_{i}}=\operatorname{vec}\left(\mathbf{H}_{n f_{i}}\right)$, which can be expressed as

$$
\mathbf{h}_{n f_{i}}=\sum_{l=1}^{L} \sum_{k=1}^{K_{l}} \mathbf{b}_{\operatorname{tr} f_{i}, l k} \alpha_{n, l k} e^{-j 2 \pi f_{i} \tau_{l}}
$$

where $\mathbf{b}_{\operatorname{tr} f_{i}, l k}$ is the steering vector of the $k_{l}$ th ray at frequency bin $f_{i}$ given as

$$
\mathbf{b}_{\operatorname{tr} f_{i}, l k}=\mathbf{A}_{\operatorname{tr} f_{i}, l k} \boldsymbol{\beta}_{l k} .
$$

Here, $\mathbf{A}_{\operatorname{tr} f_{i}, l k}$ is the steering matrix of the $k_{l}$ th ray at frequency bin $f_{i}$, which is defined as

$$
\mathbf{A}_{\mathrm{tr} f_{i}, l k}=\left(\mathbf{A}_{\mathrm{t} f_{i}, l k} \otimes \mathbf{A}_{\mathrm{r} f_{i}, l k}\right)
$$


where $\mathbf{A}_{\mathrm{t} f_{i}, l k}$ represents the steering matrix of the $k_{l}$ th ray at frequency bin $f_{i}$ in the transmitting side denoted as

$$
\mathbf{A}_{\mathrm{t} f_{i}, l k}=\mathbf{a}_{\mathrm{t} f_{i}, l k} \otimes\left[\mathbf{g}_{\mathrm{t}, l k}^{\mathrm{T}} \oplus \mathbf{A}_{\mathrm{pdt}, f_{i}, l k}^{\mathrm{T}}\right]^{\mathrm{T}} .
$$

In (19), $\boldsymbol{\beta}_{l k}=\operatorname{vec}\left(\mathbf{T}_{l k}\right)$ is a column vector. For the corresponding receiving array response, it is denoted by substituting the subscript ' $t$ ' in (21) with ' $r$ '. For compact expression, let us rewrite (18) as

$$
\mathbf{h}_{n f_{i}}=\mathbf{B}_{\mathrm{tr} f_{i}} \mathbf{F}_{f_{i}} \boldsymbol{\alpha}_{n}=\mathbf{B}_{f_{i}} \boldsymbol{\alpha}_{n}
$$

where

$$
\mathbf{B}_{\operatorname{tr} f_{i}}=\left[\mathbf{b}_{\operatorname{tr} f_{i}, 11}, \ldots, \mathbf{b}_{\operatorname{tr} f_{i}, 1 K_{1}}, \ldots, \mathbf{b}_{\operatorname{tr} f_{i}, L 1}, \ldots, \mathbf{b}_{\operatorname{tr} f_{i}, L K_{L}}\right]
$$

and $\boldsymbol{\alpha}_{n}$ is the amplitude vector for all rays in snapshot $n$ denoted as

$$
\boldsymbol{\alpha}_{n}=\left[\alpha_{n, 11}, \ldots, \alpha_{n, 1 K_{1}}, \ldots, \alpha_{n, L K_{L}}, \ldots, \alpha_{n, L K_{L}}\right]^{\mathrm{T}}
$$

In (22), $\mathbf{F}_{f_{i}}$ is the phase shifting diagonal matrix of frequency bin $f_{i}$ caused by the delay written as

$$
\mathbf{F}_{f_{i}}=\operatorname{diag}\{\underbrace{e^{-j 2 \pi f_{i} \tau_{1}}, \ldots, e^{-j 2 \pi f_{i} \tau_{1}}}_{K_{1}}, \ldots, \underbrace{e^{-j 2 \pi f_{i} \tau_{L}}, \ldots, e^{-j 2 \pi f_{i} \tau_{L}}}_{K_{L}}\} .
$$

Then, $\mathbf{h}_{n, f_{i}}$ can be estimated as

$$
\hat{\mathbf{h}}_{n f_{i}}=\operatorname{vec}\left(\mathbf{Y}_{n f_{i}} \tilde{\mathbf{S}}_{f_{i}}^{\mathrm{H}}\right) .
$$

Thus, the estimated channel response in SFP domain for all snapshots can be obtained as

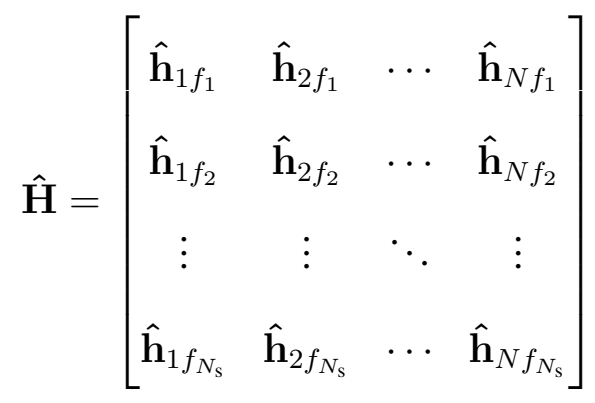

which can be further expressed as

$$
\hat{\mathbf{H}}=\mathbf{B} \Gamma+\hat{\mathbf{Z}}
$$

where $\mathbf{B}, \Gamma$ and $\hat{\mathbf{Z}}$ respectively represent steering matrix of the array, the amplitude of rays for $N$ snapshots and the noise matrix, which are given as

$$
\mathbf{B}=\left[\mathbf{B}_{f_{1}}^{\mathrm{T}}, \mathbf{B}_{f_{2}}^{\mathrm{T}}, \ldots, \mathbf{B}_{f_{N_{\mathrm{s}}}}^{\mathrm{T}}\right]^{\mathrm{T}}
$$




$$
\Gamma=\left[\boldsymbol{\alpha}_{1}, \ldots, \boldsymbol{\alpha}_{N}\right],
$$

and

$$
\hat{\mathbf{Z}}=\left[\begin{array}{ccc}
\operatorname{vec}\left(\mathbf{Z}_{1 f_{1}} \mathbf{S}_{f_{1}}^{\mathrm{H}}\right) & \cdots & \operatorname{vec}\left(\mathbf{Z}_{N f_{1}} \mathbf{S}_{f_{1}}^{\mathrm{H}}\right) \\
\vdots & \ddots & \vdots \\
\operatorname{vec}\left(\mathbf{Z}_{1 f_{N_{\mathrm{s}}}} \mathbf{S}_{f_{N_{\mathrm{s}}}}^{\mathrm{H}}\right) & \cdots & \operatorname{vec}\left(\mathbf{Z}_{N f_{N_{\mathrm{s}}}} \mathbf{S}_{f_{N_{\mathrm{s}}}}^{\mathrm{H}}\right)
\end{array}\right] .
$$

\section{Multiparameter Estimation}

\section{A. Maximum Likelihood Estimator}

The maximum likelihood (ML) estimator is considered to be an accurate estimator. The likelihood function can be written compactly as

$$
L\left(\boldsymbol{\Theta}_{\mathrm{t}}, \boldsymbol{\Theta}_{\mathrm{r}}, \boldsymbol{\Xi}\right)=\underset{\boldsymbol{\Theta}_{\mathrm{t}}, \boldsymbol{\Theta}_{\mathrm{r}}, \boldsymbol{\Xi}}{\arg \max }\left\{M_{\mathrm{t}} M_{\mathrm{r}} N_{\mathrm{s}} \ln F\left(\boldsymbol{\Theta}_{\mathrm{t}}, \boldsymbol{\Theta}_{\mathrm{r}}, \boldsymbol{\Xi}\right)\right\}
$$

where

$$
F\left(\boldsymbol{\Theta}_{\mathrm{t}}, \boldsymbol{\Theta}_{\mathrm{r}}, \boldsymbol{\Xi}\right)=\operatorname{tr}\left\{\hat{\mathbf{H}} \hat{\mathbf{H}}^{\mathrm{H}}-\mathbf{B}_{\mathrm{tr}}\left(\boldsymbol{\Theta}_{\mathrm{t}}, \boldsymbol{\Theta}_{\mathrm{r}}, \boldsymbol{\Xi}\right) \mathbf{B}_{\mathrm{tr}}^{\dagger}\left(\boldsymbol{\Theta}_{\mathrm{t}}, \boldsymbol{\Theta}_{\mathrm{r}}, \boldsymbol{\Xi}\right) \hat{\mathbf{H}} \hat{\mathbf{H}}^{\mathrm{H}}\right\} .
$$

Thus, the ML estimate of $\boldsymbol{\Theta}_{\mathrm{t}}, \boldsymbol{\Theta}_{\mathrm{r}}, \boldsymbol{\Xi}$ is obtained by maximizing $F\left(\boldsymbol{\Theta}_{\mathrm{t}}, \boldsymbol{\Theta}_{\mathrm{r}}, \boldsymbol{\Xi}\right)$. Due to the large number of impinging rays and multiple parameters, the ML estimator consumes substantial computational resources. Therefore, these multiple parameters must be estimated one after the other with much lower complexity.

\section{B. Delay and Angle Estimation}

The propagation delay of a ray is estimated with the reference antenna element. Define a selection matrix $\mathbf{J}_{\tau}=\mathbf{I}_{N_{\mathrm{s}}} \otimes \mathbf{e}_{1}^{\mathrm{T}}$, where $\mathbf{e}_{1}$ is the first column of $\mathbf{I}_{36 M_{\mathrm{r}} M_{\mathrm{t}}}$. Then, the channel response of the reference antenna element is expressed as

$$
\hat{\mathbf{H}}_{\tau}=\mathbf{J}_{\tau} \hat{\mathbf{H}} \text {. }
$$

Let $\hat{\mathbf{R}}_{\tau}=\frac{1}{N} \hat{\mathbf{H}}_{\tau} \hat{\mathbf{H}}_{\tau}^{\mathrm{H}}$ be the covariance matrix of $\hat{\mathbf{H}}_{\tau}$. The rank of $\hat{\mathbf{R}}_{\tau}$ is the number of clusters without considering the noise. The eigenvalue decomposition (EVD) of $\hat{\mathbf{R}}_{\tau}$ leads to the $N_{\mathrm{s}} \times$ $\left(N_{\mathrm{s}}-L\right)$ noise subspace $\mathbf{U}_{\mathrm{n}, \tau}$. The delay MUSIC spectrum can be expressed as

$$
P_{\tau}(\tau)=\underset{\tau}{\arg \min }\left|\mathbf{a}_{\tau}^{\mathrm{H}}(\tau) \mathbf{U}_{\mathrm{n}, \tau} \mathbf{U}_{\mathrm{n}, \tau}^{\mathrm{H}} \mathbf{a}_{\tau}(\tau)\right|
$$


where $\mathbf{a}_{\tau}$ is the steering vector in the frequency domain of the reference antenna element, which can be expressed as

$$
\mathbf{a}_{\tau}(\tau)=\left[e^{-j 2 \pi f_{1} \tau}, e^{-j 2 \pi f_{2} \tau}, \ldots, e^{-j 2 \pi f_{N_{\mathrm{s}}} \tau}\right]^{\mathrm{T}}
$$

The AoD can be estimated with the channel response related to the transmitting antennas and the reference receiving antenna elements. Define a selection matrix $\mathbf{J}_{\mathrm{t}}=\mathbf{I}_{6 N_{\mathrm{s}} M_{\mathrm{t}}} \otimes \mathbf{e}_{1}^{\mathrm{T}}$, where $\mathbf{e}_{1}$ is the first column of $\mathbf{I}_{6 M_{\mathrm{r}}}$. Then, the object channel response is given as

$$
\hat{\mathbf{H}}_{\mathrm{t}}=\mathbf{J}_{\mathrm{t}} \hat{\mathbf{H}}=\tilde{\mathbf{B}}_{\mathrm{t}} \boldsymbol{\Gamma}+\mathbf{J}_{\mathrm{t}} \hat{\mathbf{Z}}
$$

where $\tilde{\mathbf{B}}_{\mathrm{t}}$ is the steering matrix of the object channel response, i.e.,

$$
\tilde{\mathbf{B}}_{\mathrm{t}}=\left[\left(\tilde{\mathbf{B}}_{\operatorname{tr} f_{1}} \mathbf{F}_{f_{1}}\right)^{\mathrm{T}}, \ldots,\left(\tilde{\mathbf{B}}_{\operatorname{tr} f_{N_{\mathrm{s}}}} \mathbf{F}_{f_{N_{\mathrm{s}}}}\right)^{\mathrm{T}}\right]^{\mathrm{T}} .
$$

Here, $\tilde{\mathbf{B}}_{\operatorname{tr} f_{i}}$ is obtained as

$$
\tilde{\mathbf{B}}_{\operatorname{tr} f_{i}}=\mathbf{J}_{\mathrm{t}, \mathrm{ref}} \mathbf{B}_{\operatorname{tr} f_{i}}
$$

where $\mathbf{J}_{\mathrm{t}, \text { ref }}=\mathbf{I}_{6 M_{\mathrm{t}}} \otimes \mathbf{e}_{1}^{\mathrm{T}}$ and $\mathbf{e}_{1}$ is the first column of $\mathbf{I}_{6 M_{\mathrm{r}}}$. Let $\hat{\mathbf{R}}_{\mathrm{t}}=\frac{1}{N} \hat{\mathbf{H}}_{\mathrm{t}} \hat{\mathbf{H}}_{\mathrm{t}}^{\mathrm{H}}$ be the covariance matrix of $\hat{\mathbf{H}}_{\mathrm{t}}$. The rank of $\hat{\mathbf{R}}_{\mathrm{t}}$ is equal to the number of rays without considering the noise. The EVD of $\hat{\mathbf{R}}_{\mathrm{t}}$ leads to the $6 M_{\mathrm{t}} N_{\mathrm{s}} \times\left(6 M_{\mathrm{t}} N_{\mathrm{s}}-K\right)$ noise subspace $\mathbf{U}_{\mathrm{tn}}$. The AoD MUSIC spectrum can be expressed as

$$
P_{\mathrm{t}}\left(\Theta_{\mathrm{t}, l k}\right)=\underset{\Theta_{\mathrm{t}, l k}}{\arg \min } \operatorname{det}\left\{\left|\mathbf{A}_{\mathrm{t}, l k}^{\mathrm{H}}\left(\Theta_{\mathrm{t}, l k}\right) \mathbf{U}_{\mathrm{tn}} \mathbf{U}_{\mathrm{tn}}^{\mathrm{H}} \mathbf{A}_{\mathrm{t}, l k}\left(\Theta_{\mathrm{t}, l k}\right)\right|\right\} .
$$

The derivation process is shown in Appendix A.

Similarly, the AoA is estimated with the channel response related to the receiving antennas and the reference transmitting antenna element. Define a selection matrix $\mathbf{J}_{\mathrm{r}}=\mathbf{I}_{N_{\mathrm{s}}} \otimes \mathbf{e}_{1}^{\mathrm{T}} \otimes \mathbf{I}_{6 M_{\mathrm{r}}}$, where $\mathbf{e}_{1}$ is the first column of $\mathbf{I}_{6 M_{\mathrm{t}}}$. Then, the object channel response is

$$
\hat{\mathbf{H}}_{\mathrm{r}}=\mathbf{J}_{\mathrm{r}} \hat{\mathbf{H}}=\tilde{\mathbf{B}}_{\mathrm{r}} \boldsymbol{\Gamma}+\mathbf{J}_{\mathrm{r}} \hat{\mathbf{Z}}
$$

where $\tilde{\mathbf{B}}_{\mathrm{r}}$ is the steering matrix of the object channel response, i.e.,

$$
\tilde{\mathbf{B}}_{\mathrm{r}}=\left[\left(\tilde{\mathbf{B}}_{\operatorname{tr} f_{1}} \mathbf{F}_{f_{1}}\right)^{\mathrm{T}}, \ldots,\left(\tilde{\mathbf{B}}_{\operatorname{tr} f_{N_{\mathrm{s}}}} \mathbf{F}_{f_{N_{\mathrm{s}}}}\right)^{\mathrm{T}}\right]^{\mathrm{T}} .
$$

Here, $\tilde{\mathbf{B}}_{\operatorname{tr} f_{i}}$ is obtained as

$$
\tilde{\mathbf{B}}_{\mathrm{tr} f_{i}}=\mathbf{J}_{\mathrm{r}, \mathrm{ref}} \mathbf{B}_{\mathrm{tr} f_{i}}
$$




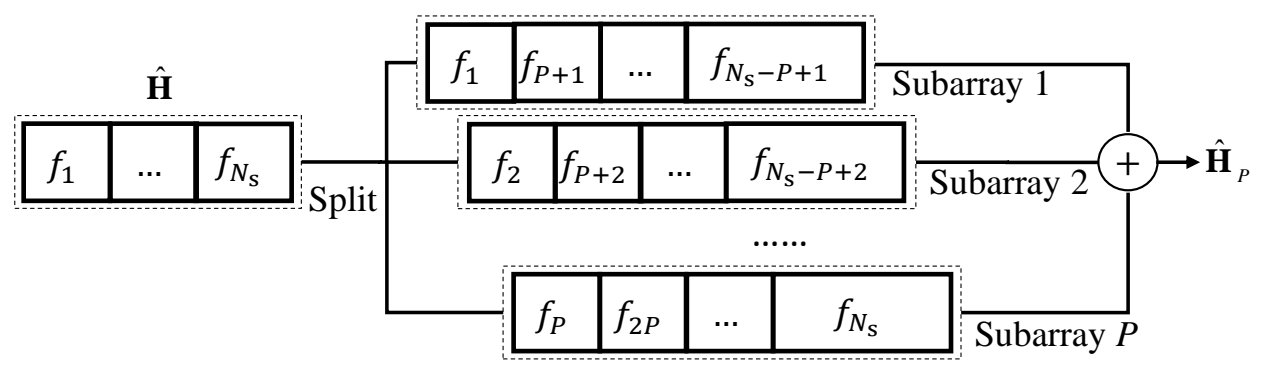

Fig. 4. Diagram of the subarray division method.

where $\mathbf{J}_{\mathrm{r}, \text { ref }}=\mathbf{e}_{1}^{\mathrm{T}} \otimes \mathbf{I}_{6 M_{\mathrm{r}}}$ and $\mathbf{e}_{1}$ is the first column of $\mathbf{I}_{6 M_{\mathrm{t}}}$. Note that $\tilde{\mathbf{B}}_{\mathrm{tr} f_{i}}$ in AoA estimation is different from that in AoD estimation, which is caused by different selection matrix $\mathbf{J}_{\mathrm{t} \text {,ref }}$ and $\mathbf{J}_{\mathrm{r}, \text { ref }}$. Let $\hat{\mathbf{R}}_{\mathrm{r}}=\frac{1}{N} \hat{\mathbf{H}}_{\mathrm{r}} \hat{\mathbf{H}}_{\mathrm{r}}^{\mathrm{H}}$ be the covariance matrix of $\hat{\mathbf{H}}_{\mathrm{r}}$. The rank of $\hat{\mathbf{R}}_{\mathrm{r}}$ is equal to the number of rays without considering the noise. The EVD of $\hat{\mathbf{R}}_{\mathrm{r}}$ leads to the $6 M_{\mathrm{r}} N_{\mathrm{s}} \times\left(6 M_{\mathrm{r}} N_{\mathrm{s}}-K\right)$ noise subspace $\mathbf{U}_{\mathrm{rn}}$. The AoA MUSIC spectrum can be expressed as

$$
P_{\mathrm{r}}\left(\Theta_{\mathrm{r}, l k}\right)=\underset{\Theta_{\mathrm{r}, l k}}{\arg \min } \operatorname{det}\left\{\left|\mathbf{A}_{\mathrm{r}}^{\mathrm{H}}\left(\Theta_{\mathrm{r}, l k}\right) \mathbf{U}_{\mathrm{rn}} \mathbf{U}_{\mathrm{rn}}^{\mathrm{H}} \mathbf{A}_{\mathrm{r}}\left(\Theta_{\mathrm{r}, l k}\right)\right|\right\} .
$$

\section{Frequency Domain Smoothing Technique}

In the application of the BEAR-based method, the FFT points are usually too large to perform EVD on the covariance matrix of the channel response. To reduce the effect of the AWGN and the computational complexity, a frequency domain smoothing preprocessing technique is proposed. The whole frequency band is divided into $P$ subfrequency bands, and each subfrequency band corresponds to a virtual subarray. Then, the channel impulse responses of all the virtual subarrays are summarized. Fig. 4 shows a diagram of the virtual subarray division. The closed-form MUSIC spectra of the AoD and AoA can be derived by means of this technique. See Appendix B for details of the derivation process.

\section{Pair Matching of $A o D$ and $A o A$}

The estimated AoD and AoA must be paired. The channel response in any frequency bin, for example, the top $36 M_{\mathrm{r}} M_{\mathrm{t}}$ rows of $\mathbf{H}$, can be used to perform pair matching. Let

$$
\mathbf{H}_{\text {pair }}=\mathbf{J}_{\text {pair }} \mathbf{H}
$$


where $\mathbf{J}_{\text {pair }}=\mathbf{e}_{1}^{\mathrm{T}} \otimes \mathbf{I}_{N_{\mathrm{s}}}$ with $\mathbf{e}_{1}$ being the first row of $\mathbf{I}_{36 M_{\mathrm{r}} M_{\mathrm{t}}}$. EVD of $\mathbf{H}_{\text {pair }}$ yields the noise subspace $\mathbf{U}_{\text {pair,n }}$.

The steering matrix for the channel response of the selected frequency bin is constructed as

$$
\mathbf{A}_{\text {pair }}\left(\hat{\Theta}_{\mathrm{r}, l k}, \hat{\Theta}_{\mathrm{t}, l k}\right)=\mathbf{A}_{\mathrm{t} f_{1}, l k}\left(\hat{\Theta}_{\mathrm{t}, l k}\right) \otimes \mathbf{A}_{\mathrm{r} f_{1}, l k}\left(\hat{\Theta}_{\mathrm{r}, l k}\right) \text {. }
$$

The aim of the pair matching of AoD and AoA is to find the AoD-AoA pair such that

$$
P_{\text {pair }}=\underset{\hat{\Theta}_{\mathrm{r}, l k}, \hat{\Theta}_{\mathrm{t}, l k}}{\arg \min } \operatorname{det}\left\{\left|\mathbf{A}_{\text {pair }}^{\mathrm{H}} \mathbf{U}_{\text {pair, } \mathrm{n}} \mathbf{U}_{\text {pair,n }}^{\mathrm{H}} \mathbf{A}_{\text {pair }}\right|\right\} .
$$

\section{E. Estimation of Initial Phases, XPR and Amplitude}

With the estimated delay and paired angles of the rays, the remaining parameters, XPR, amplitude and initial phases can be estimated subsequently. Let

$$
\mathbf{A}=\left[\begin{array}{ccc}
\mathbf{A}_{\operatorname{tr} f_{1}, 11} e^{-j 2 \pi f_{1} \tau_{1}} & \cdots & \mathbf{A}_{\operatorname{tr} f_{1}, L K_{L}} e^{-j 2 \pi f_{1} \tau_{L}} \\
\vdots & \ddots & \vdots \\
\mathbf{A}_{\operatorname{tr} f_{N_{\mathrm{s}}}, 11} e^{-j 2 \pi f_{N_{\mathrm{s}}} \tau_{1}} & \cdots & \mathbf{A}_{\operatorname{tr} f_{N_{\mathrm{s}}}, L K_{L}} e^{-j 2 \pi f_{N_{\mathrm{s}}} \tau_{L}}
\end{array}\right]
$$

The steering matrix of the array $\mathbf{B}$ can be rewritten as

$$
\mathbf{B}=\mathbf{A} \Psi
$$

where $\Psi$ is the block diagonal matrix, and can be expressed as

$$
\boldsymbol{\Psi}=\mathbf{I}_{K} \oplus\left[\boldsymbol{\beta}_{11}, \ldots, \boldsymbol{\beta}_{1 K_{1}}, \ldots, \boldsymbol{\beta}_{L 1}, \ldots, \boldsymbol{\beta}_{L K_{L}}\right]
$$

Then, $\Pi=\Psi \Gamma$ can be obtained with the estimated delay and angle as

$$
\hat{\Pi}=\hat{\mathbf{A}}^{\dagger} \hat{\mathbf{H}}
$$

To reduce the effect of the noise, sum $\hat{\Pi}$ by column to get an column vector denoted by $\mathbf{v}$, which can be written as

$$
\mathbf{v}=\left[\mathbf{v}_{11}^{\mathrm{T}}, \ldots, \mathbf{v}_{L K_{L}}^{\mathrm{T}}\right]^{\mathrm{T}}=\left[\sum_{n=1}^{N} \alpha_{n, 11} \boldsymbol{\beta}_{11}^{\mathrm{T}}, \ldots, \sum_{n=1}^{N} \alpha_{n, L K_{L}} \boldsymbol{\beta}_{L K_{L}}^{\mathrm{T}}\right]^{\mathrm{T}} .
$$

Let $\boldsymbol{\omega}$ be the initial phase vector of all rays

$$
\boldsymbol{\omega}=\left[\omega_{11}^{\mathrm{vv}}, \omega_{11}^{\mathrm{hv}}, \omega_{11}^{\mathrm{vh}}, \omega_{11}^{\mathrm{hh}}, \ldots, \omega_{L K_{L}}^{\mathrm{vv}}, \omega_{L K_{L}}^{\mathrm{hv}}, \omega_{L K_{L}}^{\mathrm{vh}}, \omega_{L K_{L}}^{\mathrm{hh}}\right]^{\mathrm{T}} .
$$


Then, the estimate of $\boldsymbol{\omega}$ can be written as

$$
\hat{\omega}=\arg \{\mathbf{v}\} .
$$

For the $k_{l}$ th ray, the XPR can be estimated as

$$
\hat{\boldsymbol{\kappa}}_{l k}=\left(\frac{\left|\mathbf{v}_{l k}(1)\right|+\left|\mathbf{v}_{l k}(4)\right|}{\left|\mathbf{v}_{l k}(2)\right|+\left|\mathbf{v}_{l k}(3)\right|}\right)^{2} .
$$

With the estimated initial phase and XPR, $\Psi$ can be reconstructed as $\hat{\Psi}$, then the amplitude of the rays for all $N$ snapshots is obtained as

$$
\hat{\Gamma}=\hat{\Psi}^{\dagger} \hat{\boldsymbol{\Pi}}
$$

\section{F. Derivation of the Cramer-Rao Lower Bound (CRLB)}

The method of CRLB derivation in [28] is used in this work. The parameters that must be estimated are $\left(\varphi_{\mathrm{t}, l k}, \theta_{\mathrm{t}, l k}, \varphi_{\mathrm{r}, l k}, \theta_{\mathrm{r}, l k}, \tau_{l}\right)$, with $l=1, \ldots, L$ and $k=1, \ldots, K_{L}$. The CRLB is given as

$$
\operatorname{CRLB}(\xi)=\frac{\sigma_{\mathrm{n}}}{2}\left\{\sum_{n=1}^{N} \operatorname{Re}\left[\boldsymbol{\Lambda}_{n}^{\mathrm{H}} \mathbf{\Upsilon}^{\mathrm{H}}\left(\mathbf{I}-\mathbf{B B}^{\dagger}\right) \boldsymbol{\Upsilon} \boldsymbol{\Lambda}_{n}\right]\right\}^{-1}
$$

where $\xi$ represents one of the parameters to be estimated, $\boldsymbol{\Lambda}_{n}=\operatorname{diag}\left\{\boldsymbol{\alpha}_{n, l k}\right\}$ and $\boldsymbol{\Upsilon}$ is given in Appendix C.

G. Implementation of the BEAR-Based Subspace Method and Comparisons with Existing Meth$o d s$

The multiparameter estimation procedure using the BEAR-based subspace method is summarized in Table I. Comparisons with existing methods are presented in Table II. The existing methods cannot resolve rays in the same cluster when the number of rays is greater than the number of antenna elements. For the genuine SAGE algorithm, the resolvable rays are not given clearly, but the low robustness caused by overlapping signals in the temporal domain and algorithm complexity limit its practical implementation. 
TABLE I

STEPS OF THE BEAR-BASEd SUbSPACE Estimator.

Steps of delay, AoD, AoA, initial phases, XPR and amplitude estimation

1: for $n=1$ to $N$ do

2: Perform FFT on the received signals of the $n$th snapshot.

3: Calculate the channel response of each frequency bin as (26).

4: Vectorize the channel response matrix as the $n$th column of (27).

5: end for

6: Calculate $\hat{\mathbf{H}}_{\tau}$ as (34), then estimate the delay using (35).

7: for $l=1$ to $L$ do

8: Calculate $\hat{\mathbf{H}}_{\mathrm{t}}$ and $\hat{\mathbf{H}}_{\mathrm{r}}$, then perform frequency smoothing to obtain

$\mathbf{H}_{\mathrm{t}, P}$ and $\mathbf{H}_{\mathrm{r}, P}$, estimate AoD using (71).

9: end for

10: Perform pair matching between AoD and AoA.

11: Estimate initial phases, XPR and amplitude with (54), (55) and (56).

TABLE II

Comparisons With Methods in the References.

\begin{tabular}{|c|c|c|}
\hline Method & Estimated parameters & Resolvable rays in one cluster \\
\hline JSTPE [19] & Delay, AAoA & $6 M_{\mathrm{r}}-1$ \\
\hline SAGE [17] & Delay, AAoA, Amplitude & - \\
\hline MAPS [18] & Delay, 2D AoA & $6 M_{\mathrm{r}}-1$ \\
\hline Proposed Method & $\begin{array}{c}\text { Delay, 2D AoA, 2D AoD, XPR } \\
\text { Amplitude, Initial Phases }\end{array}$ & $6 M_{\mathrm{r}} N_{\mathrm{s}} / P-1$ \\
\hline
\end{tabular}


TABLE III

COMPLEXITY ANALYSIS OF THE PROPOSED METHOD.

\begin{tabular}{|c|c|}
\hline Item & Complexity \\
\hline FFT for the received signal & $O\left(6 N L_{\mathrm{seq}} N_{\mathrm{s}} M_{\mathrm{r}} \log \left(6 N_{\mathrm{s}} M_{\mathrm{r}}\right)\right)$ \\
\hline EVD of $\mathbf{H}_{\tau}$ & $O\left(N_{\mathrm{s}}^{3}\right)$ \\
\hline MUSIC delay searching & $O\left(L W_{\tau} N_{\mathrm{s}}^{2}\right)$ \\
\hline EVD of $\mathbf{H}_{\mathrm{r}}$ & $O\left(216 M_{\mathrm{r}}^{3} M_{f}^{3}\right)$ \\
\hline EVD of $\mathbf{H}_{\mathrm{t}}$ & $O\left(216 M_{\mathrm{t}}^{3} M_{f}^{3}\right)$ \\
\hline MUSIC AoA searching & $O\left(48 L W_{\mathrm{AoA}} M_{\mathrm{r}} M_{f}\left(3 M_{\mathrm{r}} M_{f}+2\right)\right)$ \\
\hline MUSIC AoD searching & $O\left(48 L W_{\mathrm{AoD}} M_{\mathrm{t}} M_{f}\left(3 M_{\mathrm{t}} M_{f}+2\right)\right)$ \\
\hline
\end{tabular}

\section{H. Complexity Analysis}

The computational complexity of the proposed method is calculated in terms of complex multiplications. To reduce computational burden, the FFT is used to replace the DFT. The complexity of the FFT for a $M \times N$ matrix is $M N \log (M N)$. The number of computations for an EVD of $M \times M$ matrix is $O\left(M^{3}\right)$. Let $M_{f}=N_{\mathrm{s}} / P$ be the length of the split subarray in the frequency domain. In Table III, the complexity of estimating the parameters for all $K$ rays is shown in detail where $W_{\tau}, W_{A o D}$ and $W_{A o A}$ represent the number of rank-deficient MUSIC search times, $W_{\tau}$ is the one-dimensional search times, which is inversely proportional to the search step, and $W_{A o D}$ and $W_{A o A}$ are 2D search times, which are inversely proportional to the square of the search step. The AoD and AoA estimation is based on the delay of clusters. Thus, the searching progress need to be performed $L$ times.

\section{Numerical Simulations And AnAlysis}

\section{A. Simulation Setup}

In this section, simulations are performed to evaluate the performance of the proposed BEARbased method. Multipath propagation channels with additive white Gaussian noise are simulated. Two clusters are generated in the simulation. Each cluster contains 14 rays. The transmitting antenna array and receiving antenna array are equipped with two EMVAs (12 antenna elements), 
respectively. The number of rays is larger than the number of antenna array elements. The carrier frequency is set to be $28 \mathrm{GHz}$, the interelement spacing is larger than half of the carrier wavelength, and the bandwidth of the signals is $500 \mathrm{MHz}$. The length of the subsequence is set to $N_{\mathrm{s}}=1024$ code width, which is equal to the FFT points. The transmitting polarization of the rays is assumed to be known in the channel sounding system and can be controlled by the weighting vector $\mathbf{w}$. The number of snapshots is set to 100 , which is larger than the number of rays.

In the simulation, accurate performance of the proposed BEAR-based method is achieved under different SNRs and array sizes. To evaluate the estimation accuracy, the root mean square error (RMSE) of the angle estimation is given as

$$
\mathrm{RMSE}=\sqrt{\frac{1}{K Q} \sum_{q=1}^{Q} \sum_{l=1}^{L} \sum_{k=1}^{K_{l}}\left(\hat{\xi}_{l k, q}-\xi_{l k, q}\right)^{2}}
$$

where $Q$ is the number of experiments and $\xi$ represents the azimuth or the elevation.

\section{B. Parameter Estimation with Different SNRs}

The delays of the two clusters are set to $\tau=[100,110]$ ns, and the transmitting wave is set to be vertical polarized. The normalized MUSIC delay spectrum under different SNRs is shown in Fig. 5.

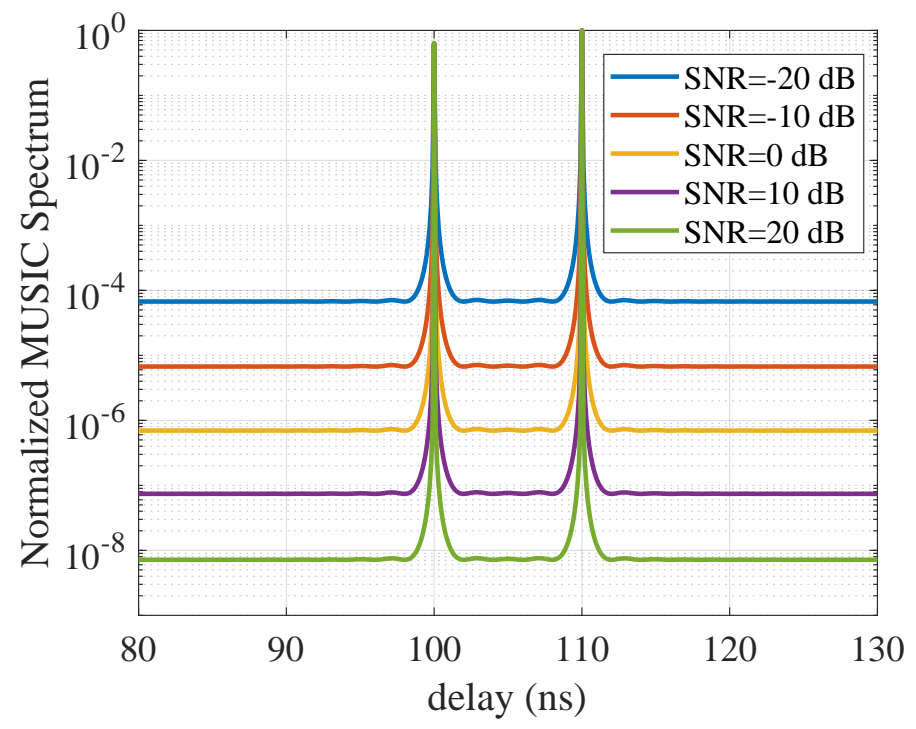

Fig. 5. The normalized MUSIC delay spectrum with different SNRs. 


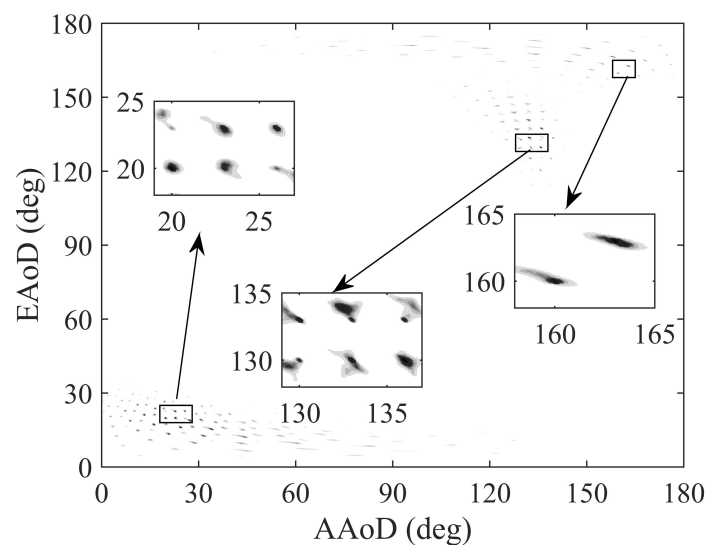

(a)

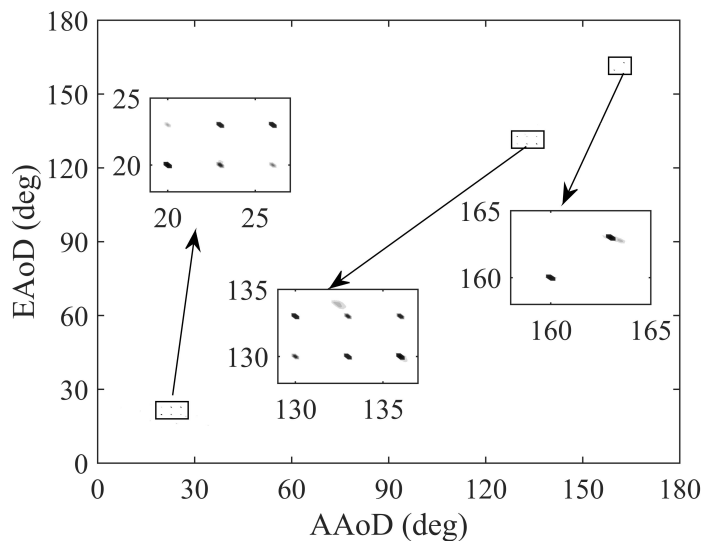

(c)

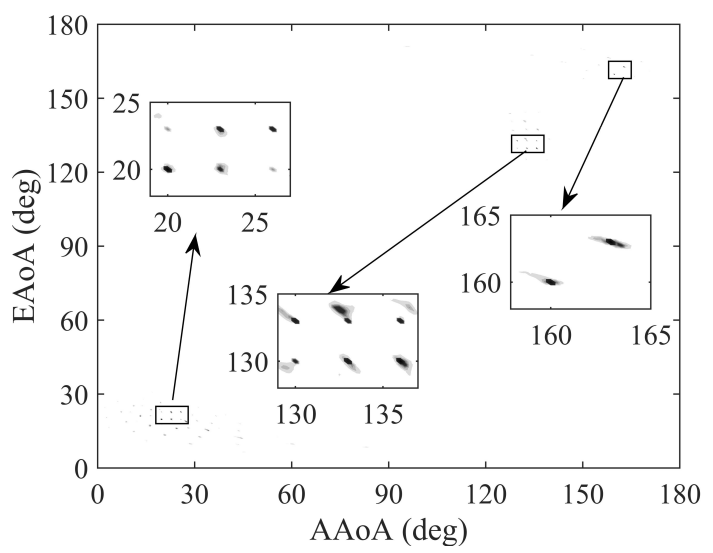

(e)

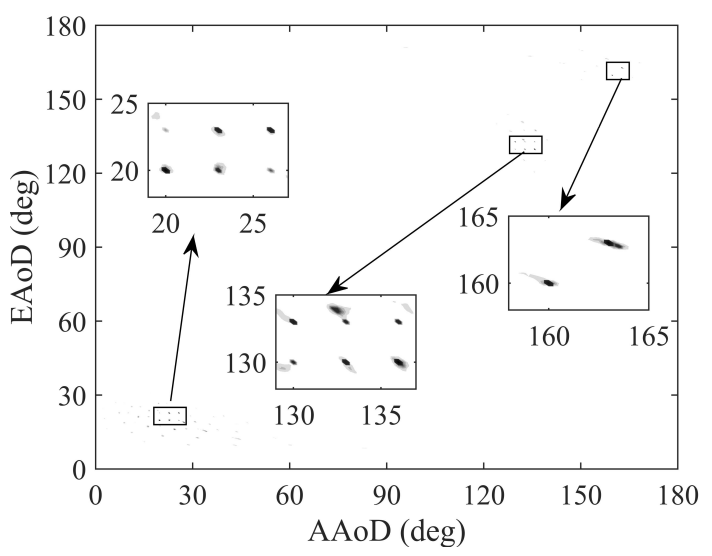

(b)

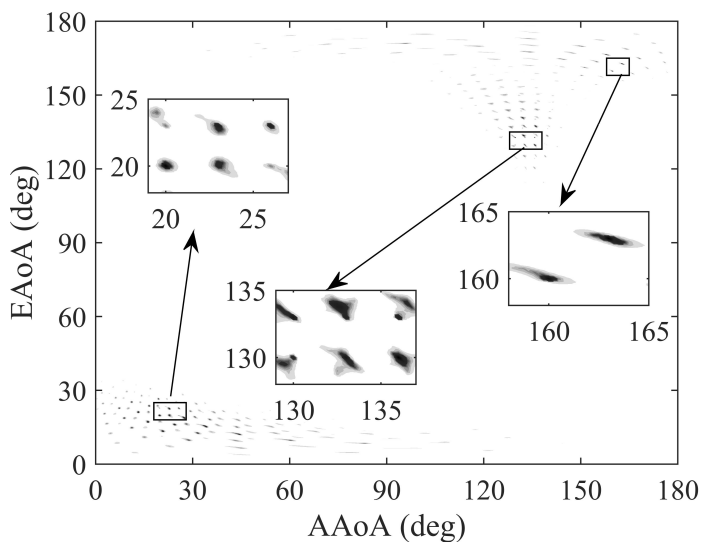

(d)

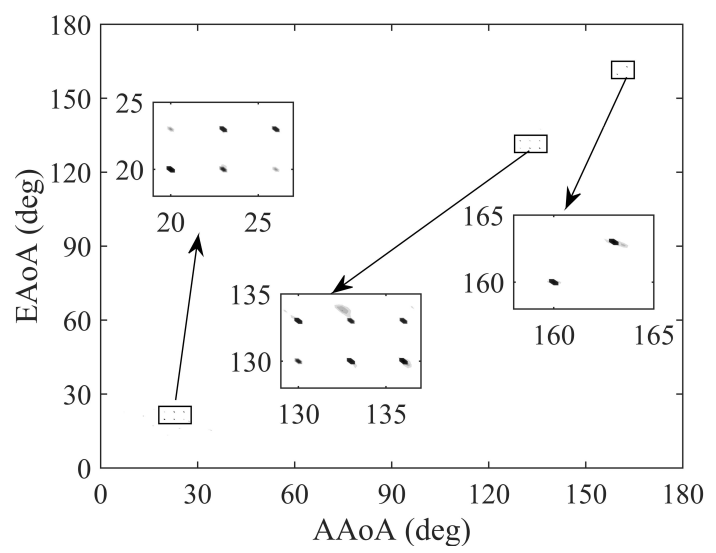

(f)

Fig. 6. The AoD and AoA MUSIC spectra with different SNRs: (a) AoD, SNR = 0 dB; (b) AoD, SNR = 5 dB; (c) AoD, $\mathrm{SNR}=10 \mathrm{~dB}$; (d) AoA, SNR = $0 \mathrm{~dB}$; (e) AoA, SNR = $5 \mathrm{~dB}$; (f) AoA, SNR = $10 \mathrm{~dB}$. 


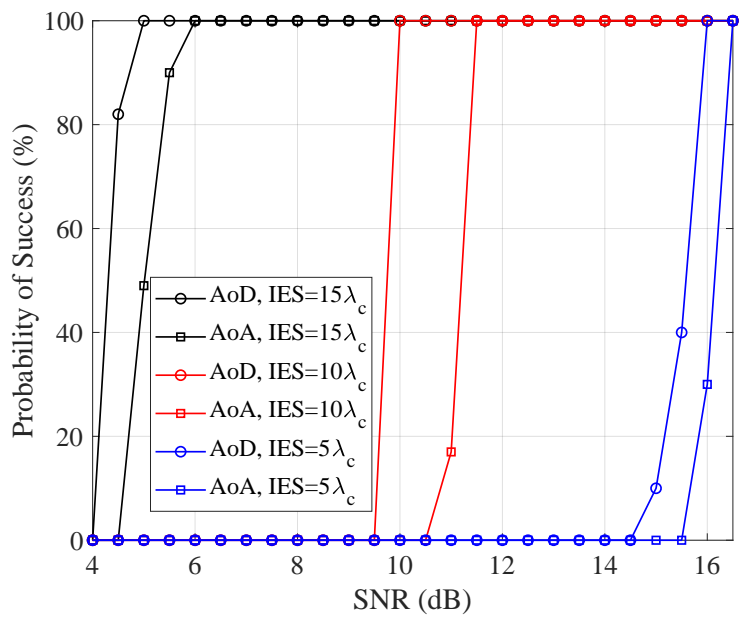

Fig. 7. The probability of success for AoD and AoA with different SNRs.

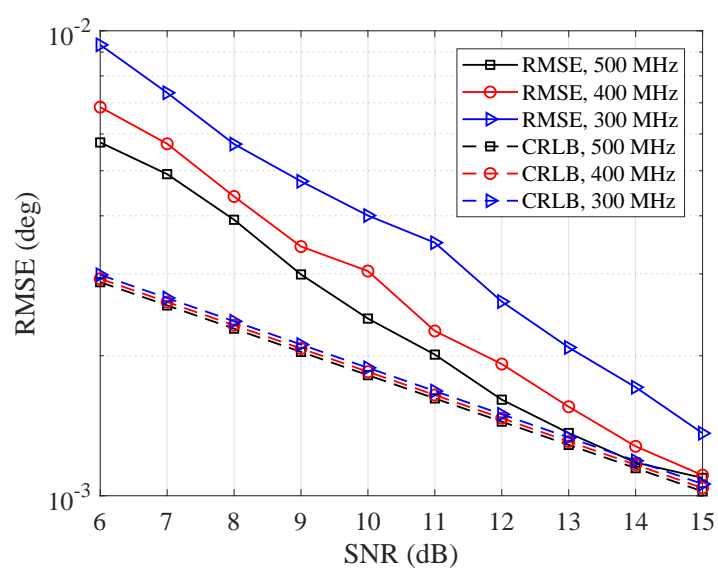

(a)

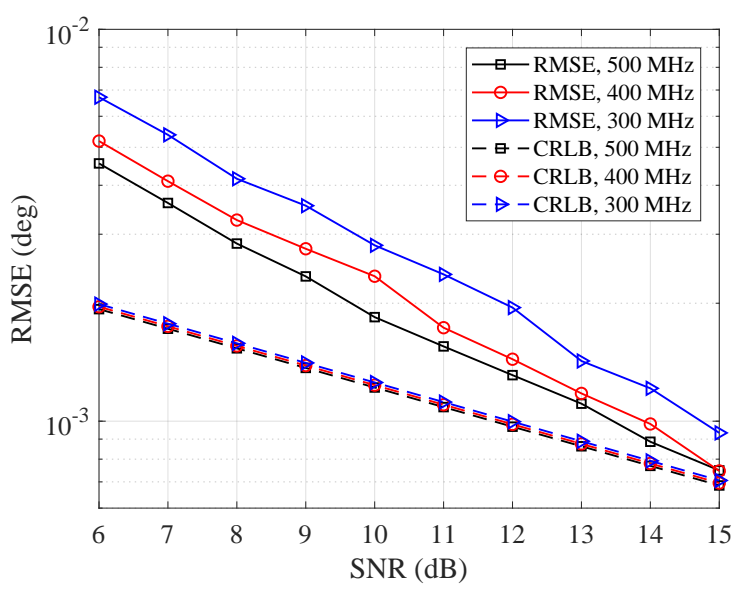

(b)

Fig. 8. The RMSE of AoD estimation under different SNRs with three different interelement spacings. (a) AAoD; (b) EAoD.

Delays can be accurately estimated even under very low SNR conditions. The AoA and AoD estimations are based on the delay estimation results, so accurate delay estimation is essential. The AoD and AoA MUSIC spectra under different SNRs are given in Fig. 6. The rays in one cluster usually result from the same scatter or scatters with nearby position. Therefore, the angles are distributed in a small scope. Furthermore, some of the rays in one cluster may have the same azimuth or elevation angle. In the simulation of Fig. 6, the azimuth and the elevation of some rays are concentrated within a small degree range. The angle range of the rays in one cluster is $[0,180]$ degrees. The proposed method can estimate the AoD and AoA accurately when the 


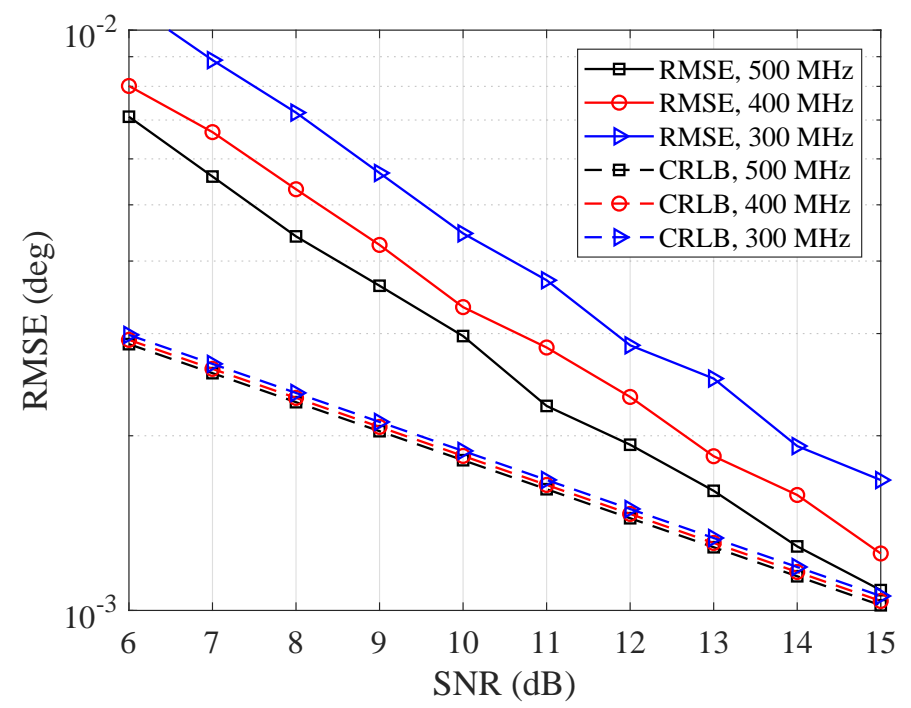

(a)

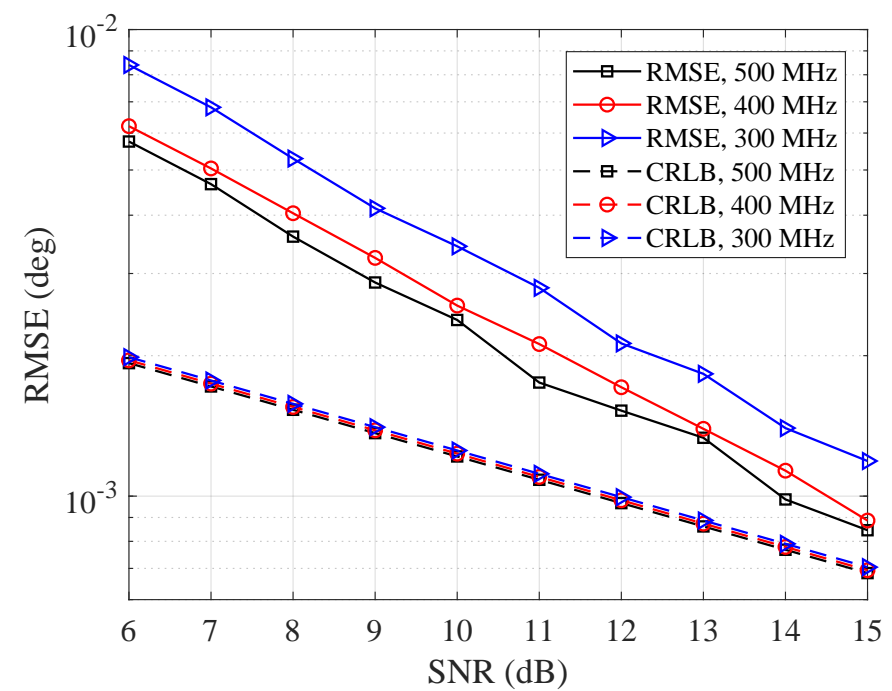

(b)

Fig. 9. The RMSE of AoA estimation under different SNRs with three different interelement spacings. (a) AAoA; (b) EAoA.

number of rays in one cluster is larger than the number of antenna elements, even if some of the rays have the same azimuths or elevations. As the SNR increases, the peaks of the rays become more clear. The angles can be estimated unambiguously when the space between two antenna elements is larger than half the carrier wavelength, which is different from the traditional methods.

Fig. 7 shows the probability of success for the AoD and AoA estimation. The interelement 


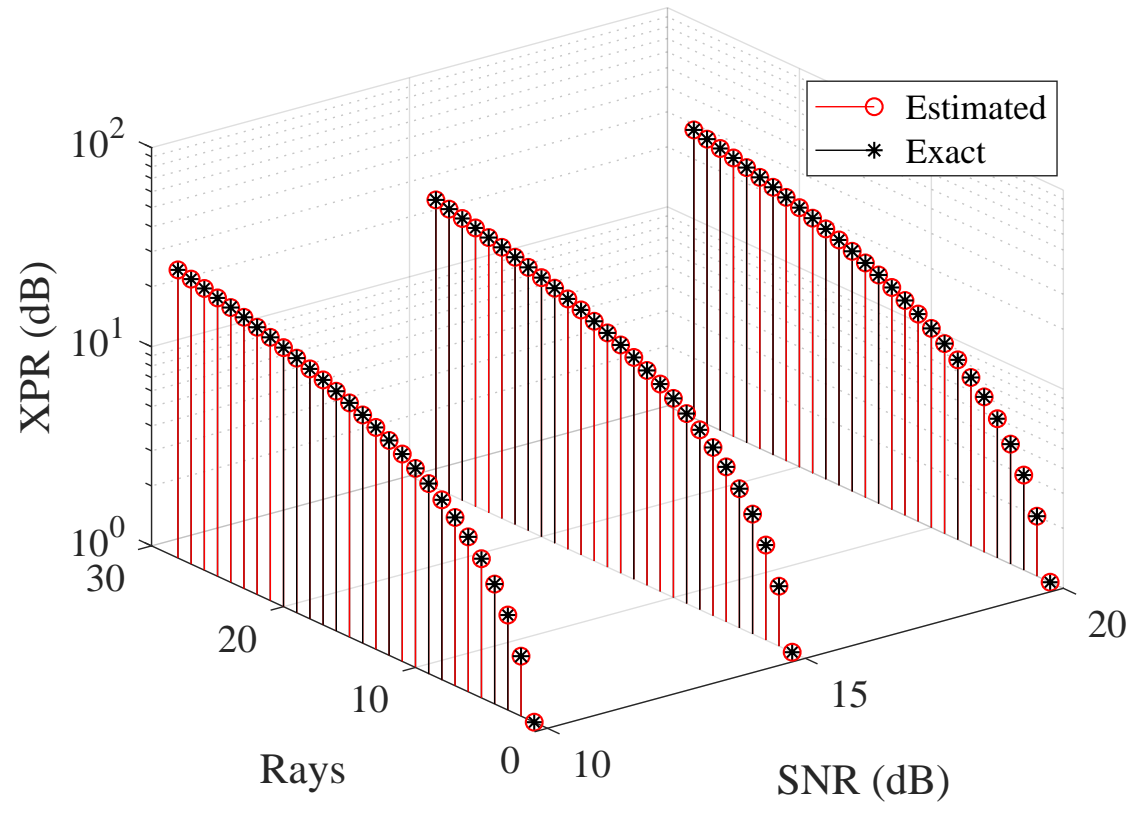

Fig. 10. The XPR estimation results under different SNRs.

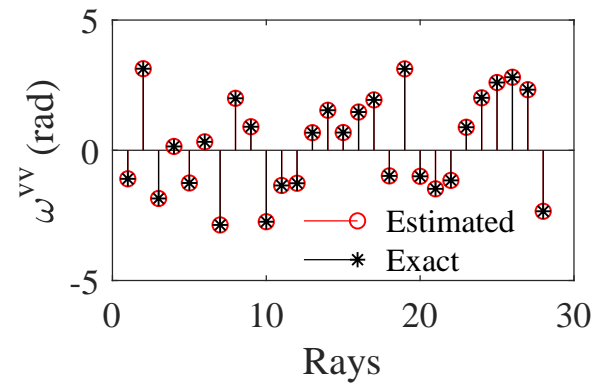

(a)

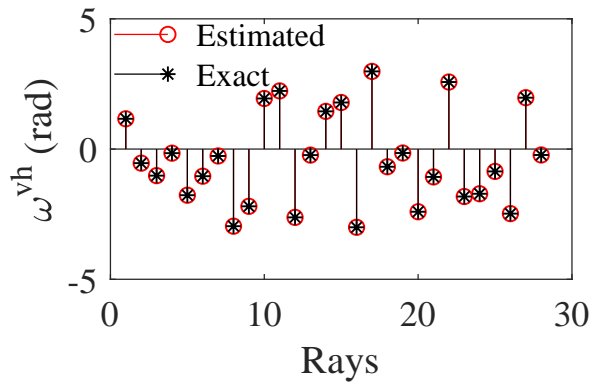

(c)

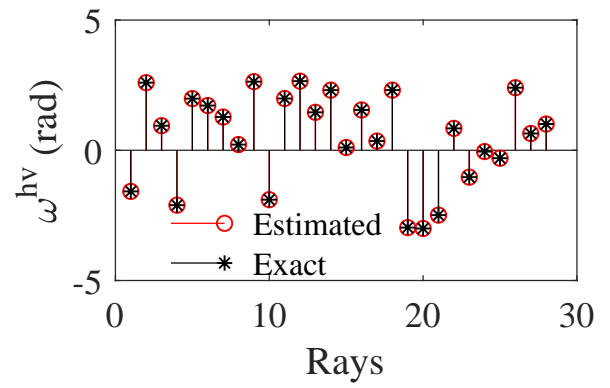

(b)

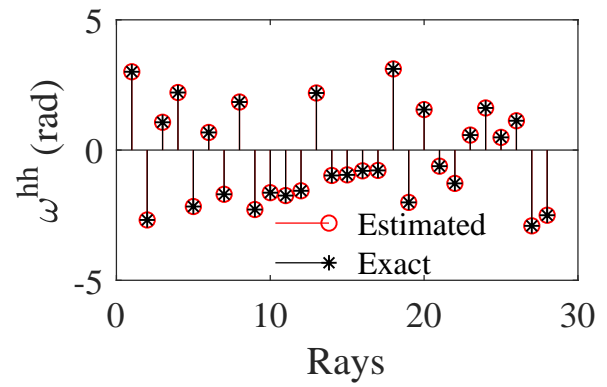

(d)

Fig. 11. The initial phases estimation results with $\mathrm{SNR}=10 \mathrm{~dB}$ for (a) $\omega^{\mathrm{vv}}$, (b) $\omega^{\mathrm{hv}}$, (c) $\omega^{\mathrm{vh}}$, and (d) $\omega^{\mathrm{hh}}$. 
spacing is $15 \lambda_{\mathrm{c}}$ or $10 \lambda_{\mathrm{c}}$. The condition for judging the success of the estimation is that the sum of the RMSE of the azimuth angle and the elevation angle is less than 1 degree. The probability of success is very close for AoD and AoA estimation under the same antenna element space. For larger antenna element space, the probability of success approaches $100 \%$ under a lower SNR.

Fig. 8 gives the RMSE of the estimated AAoD and EAoD with different bandwidth. Fig. 9 gives the RMSE of the estimated AAoA and EAoA with different bandwidth. The results show that the RMSE is affected by the bandwidth. At the same SNR, a wider signal bandwidth leads to better RMSE performance. The experiments are performed one hundred times to reduce the uncertainty. The CRLB of each situation is given for comparison. The simulation results show that the RMSEs of the proposed method are close to those of the CRLB as the SNR increases. The XPR estimation result of each ray in all clusters, given in Fig. 10, is accurately estimated. In the simulation, there are two clusters with 14 rays in each cluster. Fig. 11 gives the estimation results of the four initial phases under $10 \mathrm{~dB}$ SNR. Fig. 12 gives the estimation results of the amplitude of all the rays in $N$ snapshot. In the simulation, most of the rays are set with weak power fluctuated in a small range and only a few rays are set with strong power. This situation setting is usually practical in the real radio propagation environment. The results show that the initial phases and the amplitude can be estimated accurately under $10 \mathrm{~dB}$ SNR.

The minimum SNR required to extract all the rays successfully under different $L_{\text {seq }}$ with different array sizes is plotted in Fig. 13. The required minimum SNR decreases as $L_{\text {seq }}$ and array size increases because the increase in $L_{\text {seq }}$ can suppress the influence of noise and the increase in the array size can enlarge the array aperture.

\section{Conclusions}

A novel BEAR-based method has been proposed to estimate the delay, azimuth and elevation angles of arrival and departure, amplitude, XPR and initial phase with EMVAA for multipolarized channel measurements. By extending the array response in the SFP domain, the proposed method can estimate a large number of rays in one cluster and is not limited by the number of antenna elements. The proposed method works even if the rays have similar azimuths and elevations. The $\mathrm{XPR}$, which is usually obtained coarsely based on the power ratio of each polarized antenna elements, can be estimated accurately with the proposed method. In addition, the four initial 


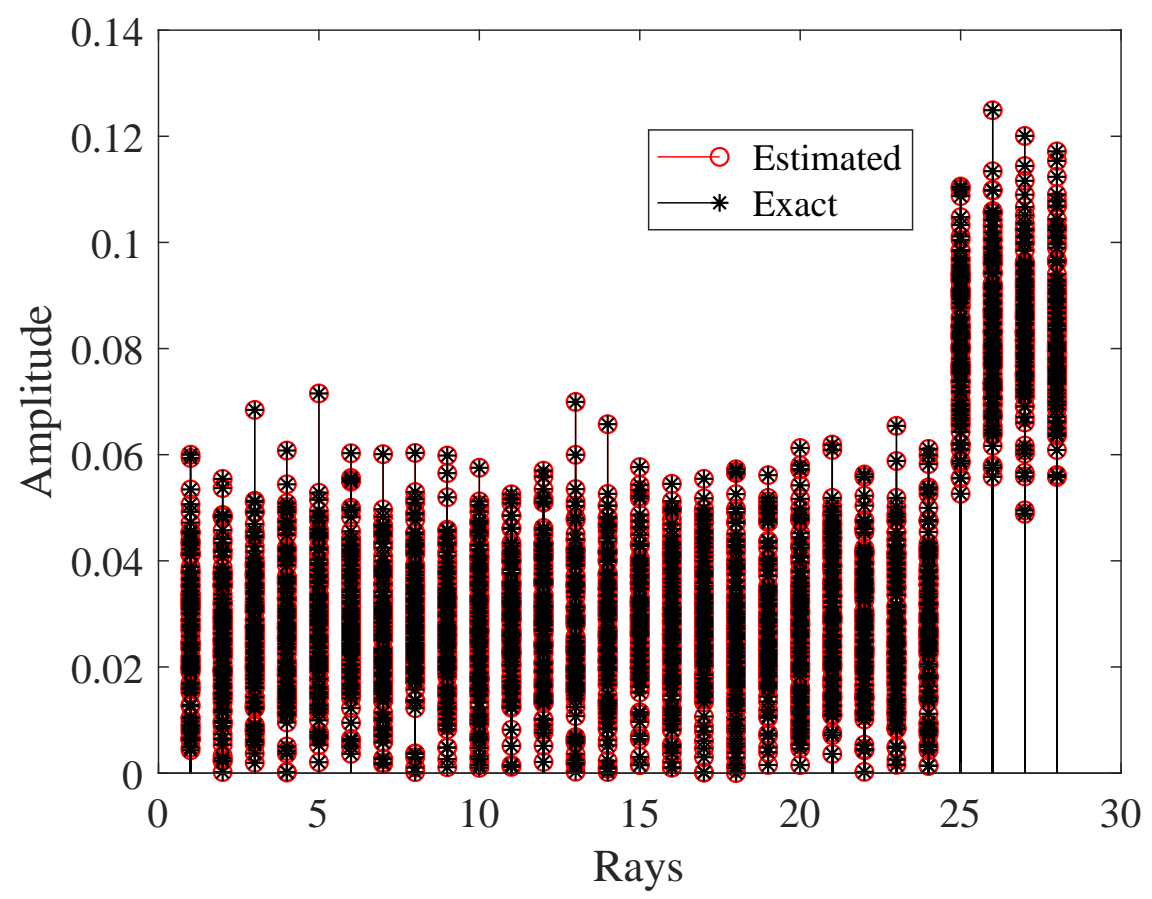

Fig. 12. The amplitude estimation results when $\mathrm{SNR}=10 \mathrm{~dB}$.

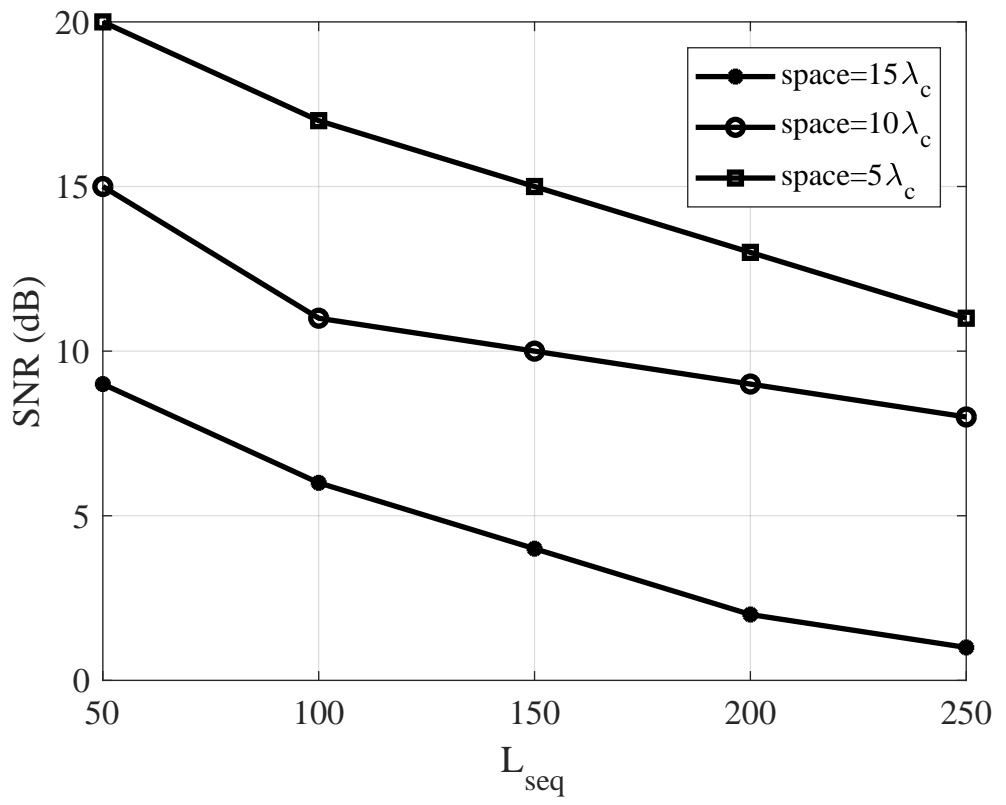

Fig. 13. The relationship between the minimum required SNR and $L_{\text {seq }}$ with a $100 \%$ probability of success. 
phases of different polarization combinations are estimated accurately. The interelement spacing can be larger than half the carrier wavelength without angle ambiguity in the angle estimation. Thus, mutual coupling is effectively suppressed. The simulation results have shown that larger bandwidth leads to a smaller RMSE of the AoA and AoD. Moreover, larger interelement spacing leads to smaller required SNR. The proposed method is a promising candidate for multiparameter estimation in future multipolarized channel measurements.

\section{APPENDIX A}

\section{DERIVATION OF THE RANK-DEFICIENT MUSIC SPECTRUM OF AOD}

The steering matrix of the channel response matrix related to the transmitting antenna array and the reference receiving antenna element is $\tilde{\mathbf{B}}_{\mathrm{t}}$, which is related to $\tilde{\mathbf{B}}_{\operatorname{tr} f_{i}}$ as (38). According to (19) and (39), the column of $\tilde{\mathbf{B}}_{\operatorname{tr} f_{i}}$ can be rewritten as

$$
\tilde{\mathbf{b}}_{\mathrm{tr} f_{i}, l k}=\left(\mathbf{A}_{\mathrm{t} f_{i}, l k} \otimes \mathbf{1}_{1,2}\right) \boldsymbol{\beta}_{l k} .
$$

Let $\mathbf{A}_{\mathrm{t}, l k}$ be

$$
\mathbf{A}_{\mathrm{t}, l k}=\left[e^{-j 2 \pi f_{1} \tau_{l}} \mathbf{A}_{\mathrm{t} f_{1}, l k}^{\mathrm{T}}, \ldots, e^{-j 2 \pi f_{N_{\mathrm{s}}} \tau_{l}} \mathbf{A}_{\mathrm{t} f_{N_{\mathrm{s}}}, l k}^{\mathrm{T}}\right]^{\mathrm{T}} .
$$

Then, the column of $\tilde{\mathbf{B}}_{\mathrm{t}}$ can be written as

$$
\tilde{\mathbf{b}}_{\mathrm{t}, l k}=\left(\mathbf{A}_{\mathrm{t}, l k} \otimes \mathbf{1}_{1,2}\right) \boldsymbol{\beta}_{l k}
$$

In order to decrease the MUSIC searching dimension, rank-deficient MUSIC technique is applied in AoD estimation [29]. Let $\mathbf{A}_{\mathrm{AoD}, l k}=\left(\mathbf{A}_{\mathrm{t}, l k} \otimes \mathbf{1}_{1,2}\right)$, the spectrum can be written as

$$
P_{t}\left(\Theta_{\mathrm{t}, l k}\right)=\arg \min _{\Theta_{\mathrm{t}, l k}} \operatorname{det}\left\{\left|\mathbf{A}_{\mathrm{AoD}, l k}^{\mathrm{H}}\left(\Theta_{\mathrm{t}, l k}\right) \mathbf{U}_{\mathrm{tn}} \mathbf{U}_{\mathrm{tn}}^{\mathrm{H}} \mathbf{A}_{\mathrm{AoD}, l k}\left(\Theta_{\mathrm{t}, l k}\right)\right|\right\} .
$$

Due to the fact that the columns of $\mathbf{A}_{\mathrm{AoD}, l k}$ are linear dependent with that of $\mathbf{A}_{\mathrm{t}, l k}$, (62) can be simplified as

$$
P_{t}\left(\Theta_{\mathrm{t}, l k}\right)=\arg \min _{\Theta_{\mathrm{t}, l k}} \operatorname{det}\left\{\left|\mathbf{A}_{\mathrm{t}, l k}^{\mathrm{H}}\left(\Theta_{\mathrm{t}, l k}\right) \mathbf{U}_{\mathrm{tn}} \mathbf{U}_{\mathrm{tn}}^{\mathrm{H}} \mathbf{A}_{\mathrm{t}, l k}\left(\Theta_{\mathrm{t}, l k}\right)\right|\right\}
$$




\section{APPENDIX B}

\section{Derivation of Frequency Domain SMOothing MUSIC SPECTRUm of AoD AND AoA}

Let us define a selection matrix for AoD estimation

$$
\mathbf{J}_{\mathrm{t}, \mathrm{sm}, i}=\mathbf{I}_{N_{\mathrm{s}} / P} \otimes \mathbf{e}_{i}^{\mathrm{T}} \otimes \mathbf{I}_{6 M_{\mathrm{t}}}
$$

where $P$ is the number of virtual subarrays, and is divisible by $N_{\mathrm{s}}$, and $\mathbf{e}_{i}$ is the $i$ th column of $\mathbf{I}_{P}$.

Let $\Phi_{\mathrm{t}, p}$ be the phase shifting matrix of adjacent frequency bins of the transmitting antenna elements, can be expressed as

$$
\boldsymbol{\Phi}_{\mathrm{t}, p}= \begin{cases}\mathbf{1}_{6 M_{\mathrm{t}} N_{\mathrm{s}} / P \times K}, & p=0, \\ {\left[\phi_{\mathrm{t}, 11}, \ldots, \phi_{\mathrm{t}, 1 K_{1}}, \ldots, \phi_{\mathrm{t}, L 1}, \ldots, \phi_{\mathrm{t}, L K_{L}}\right],} & p=1, \\ \boldsymbol{\Phi}_{\mathrm{t}, p-1} \odot \boldsymbol{\Phi}_{\mathrm{t}, 1}, & p>1,\end{cases}
$$

where $\phi_{\mathrm{t}, l k}$ is the column of $\boldsymbol{\Phi}_{\mathrm{t}, 1}$, and can be written as

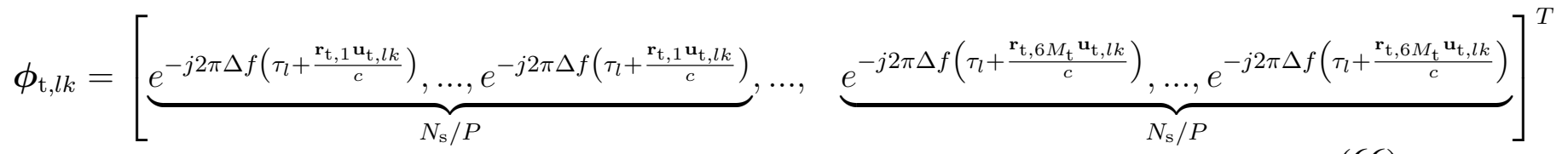

with $\mathbf{r}_{\mathrm{t}, i}$ being the coordinate of the $i$ th transmitting element and $i=1, \ldots, 6 M_{\mathrm{t}}$. Then, let us reconstruct (37) as

$$
\begin{aligned}
\hat{\mathbf{H}}_{\mathrm{t}, P} & =\frac{1}{P} \sum_{i=1}^{P} \mathbf{J}_{\mathrm{t}, \mathrm{sm}, i} \hat{\mathbf{H}}_{\mathrm{t}} \\
& =\frac{1}{P} \tilde{\mathbf{B}}_{\mathrm{t}, \mathrm{sm}} \odot \boldsymbol{\Sigma}_{\mathrm{t}} \boldsymbol{\Gamma}+\frac{1}{P} \sum_{i=1}^{P} \mathbf{J}_{\mathrm{t}, \mathrm{sm}, i} \mathbf{J}_{\mathrm{t}} \mathbf{Z}
\end{aligned}
$$

where

$$
\begin{gathered}
\tilde{\mathbf{B}}_{\mathrm{t}, \mathrm{sm}}=\mathbf{J}_{\mathrm{t}, \mathrm{sm}, 1} \mathbf{J}_{\mathrm{t}} \mathbf{B}_{\mathrm{tr}}, \\
\boldsymbol{\Sigma}_{\mathrm{t}}=\left(\boldsymbol{\Phi}_{\mathrm{t}, 0}+\boldsymbol{\Phi}_{\mathrm{t}, 1}+\ldots+\boldsymbol{\Phi}_{\mathrm{t}, P-1}\right) .
\end{gathered}
$$

The covariance matrix of $\hat{\mathbf{H}}_{\mathrm{t}, P}$ is

$$
\mathbf{R}_{\mathrm{t}, P}=\frac{P}{6 M_{\mathrm{t}} N_{\mathrm{s}}} \hat{\mathbf{H}}_{\mathrm{t}, P} \hat{\mathbf{H}}_{\mathrm{t}, P}^{\mathrm{H}} .
$$


EVD of $\mathbf{R}_{\mathrm{t}, P}$ leads to the $6 M_{\mathrm{t}} N_{\mathrm{s}} / P \times\left(6 M_{\mathrm{t}} N_{\mathrm{s}} / P-K\right)$ noise subspace $\mathbf{U}_{P \mathrm{tn}}$. The smoothed AoD MUSIC spectrum can be expressed as

$$
\bar{P}_{\mathrm{t}}\left(\Theta_{\mathrm{t}, l k}\right)=\underset{\Theta_{\mathrm{t}, l k}}{\arg \min } \operatorname{det}\left\{\left|\overline{\mathbf{A}}_{\mathrm{t}}^{\mathrm{H}}\left(\Theta_{\mathrm{t}, l k}\right) \mathbf{U}_{P \operatorname{tn}} \mathbf{U}_{P \operatorname{tn}}^{\mathrm{H}} \overline{\mathbf{A}}_{\mathrm{t}}\left(\Theta_{\mathrm{t}, l k}\right)\right|\right\}
$$

where the steering matrix $\overline{\mathbf{A}}_{\mathrm{t}}\left(\Theta_{\mathrm{t}, l k}\right)$ is given as

$$
\overline{\mathbf{A}}_{\mathrm{t}}\left(\Theta_{\mathrm{t}, l k}\right)=\mathbf{J}_{\mathrm{t}, \mathrm{sm}, 1} \mathbf{A}_{\mathrm{t}}\left(\Theta_{\mathrm{t}, l k}\right) \odot\left[\boldsymbol{\vartheta}\left(\Theta_{\mathrm{t}, l k}\right), \boldsymbol{\vartheta}\left(\Theta_{\mathrm{t}, l k}\right)\right]
$$

with $\vartheta$ representing the column expression of $\Sigma_{t}$.

\section{Appendix C \\ DERIVATION OF CRLB}

Matrix $\Upsilon$ is the derivative of $\mathbf{B}$ defined as

$$
\boldsymbol{\Upsilon}=\frac{\partial \mathbf{B}}{\partial \boldsymbol{\xi}}=\left[\begin{array}{lll}
\frac{\partial \mathbf{b}_{11}}{\partial \xi_{11}} & \ldots & \frac{\partial \mathbf{b}_{L K_{L}}}{\partial \xi_{L K_{L}}}
\end{array}\right]
$$

where $\mathbf{b}$ is the column of $\mathbf{B}$ given as

$$
\mathbf{b}_{l k}=\left[\mathbf{b}_{\operatorname{tr} f_{1}, l k} e^{-j 2 \pi f_{1} \tau_{l}}, \ldots, \mathbf{b}_{\operatorname{tr} f_{N_{\mathrm{s}}}, l k} e^{-j 2 \pi f_{N_{\mathrm{s}}} \tau_{l}}\right]
$$

Note that the antenna pattern is discrete in the space domain and cannot be differentiated. Without loss of generality, it is regarded as a unit gain in the calculation progress. $\xi$ refers to the estimated parameters. The details of the derivation process are similar to those of [28] and are not presented.

\section{REFERENCES}

[1] T. S. Rappaport et al., "Wideband millimeter-wave propagation measurements and channel models for future wireless communication system design,” IEEE Trans. Commun., vol. 63, no. 9, pp. 3029-3056, 2015.

[2] C.-X. Wang et al., "A survey of 5G channel measurements and models," IEEE Commun. Surv. Tut., vol. 20, no. 4, pp. 3142-3168, 2018.

[3] 3GPP, "Study on channel model for frequencies from 0.5 to $100 \mathrm{GHz}$," 3rd Generation Partnership Project (3GPP), TR 38.901 V15.0.0, Tech. Rep., June 2018.

[4] — - "Study on channel model for frequency spectrum above $6 \mathrm{GHz}$," 3rd Generation Partnership Project (3GPP), TR 38.900 V15.0.0, Tech. Rep., June 2018.

[5] J. Zhang, C. Pan, F. Pei, G. Liu, and X. Cheng, "Three-dimensional fading channel models: A survey of elevation angle research," IEEE Commun. Mag., vol. 52, no. 6, pp. 218-226, 2014.

[6] D. Piao and Y. Wang, "Experimental evaluation of the tri-polarized MIMO channel properties based on a compact multimode antenna," IEEE Access, vol. 7, pp. 67 807-67 817, 2019. 
[7] D. Piao, "Characteristics of the hexapolarized MIMO channel over free-space and three non-free-space scenarios," IEEE Trans. Wirel. Commun., vol. 12, no. 8, pp. 4174-4182, 2013.

[8] M. R. Andrews, P. P. Mitra, and R. DeCarvalho, "Tripling the capacity of wireless communications using electromagnetic polarization," Nature, vol. 409, no. 6818, pp. 316-318, 2001.

[9] M. Dao, V. Nguyen, Y. Im, S. Park, and G. Yoon, “3D polarized channel modeling and performance comparison of MIMO antenna configurations with different polarizations," IEEE Trans. Antennas Propag., vol. 59, no. 7, pp. 2672-2682, 2011.

[10] Y. He, X. Cheng, and G. L. Stuber, "On polarization channel modeling," IEEE Wirel. Commun., vol. 23, no. 1, pp. 80-86, 2016.

[11] M. Coldrey, "Modeling and capacity of polarized MIMO channels," in VTC Spring 2008 - IEEE Vehicular Technology Conference, 2008, pp. 440-444.

[12] A. S. Y. Poon and D. N. C. Tse, "Degree-of-freedom gain from using polarimetric antenna elements," IEEE Trans. Inf. Theory, vol. 57, no. 9, pp. 5695-5709, 2011.

[13] X. Wang, L. Wan, M. Huang, C. Shen, and K. Zhang, "Polarization channel estimation for circular and non-circular signals in massive MIMO systems," IEEE J. Sel. Top. Signal Process., vol. 13, no. 5, pp. 1001-1016, 2019.

[14] Y. Bresler and A. Macovski, "Exact maximum likelihood parameter estimation of superimposed exponential signals in noise," IEEE Trans. Acoust. Speech Signal Process., vol. 34, no. 5, pp. 1081-1089, 1986.

[15] Yung-Yi Wang, Jiunn-Tsair Chen, and Wen-Hsien Fang, “TST-MUSIC for joint DOA-delay estimation,” IEEE Trans. Signal Process., vol. 49, no. 4, pp. 721-729, 2001.

[16] M. C. Vanderveen et al., "Estimation of multipath parameters in wireless communications," IEEE Trans. Signal Process., vol. 46, no. 3, pp. 682-690, 1997.

[17] B. H. Fleury et al., "Channel parameter estimation in mobile radio environments using the SAGE algorithm," IEEE J. Sel. Areas Commun., vol. 17, no. 3, pp. 434-450, 1999.

[18] R. Zhang, S. Wang, X. Lu, W. Duan, and L. Cai, "Two-dimensional DoA estimation for multipath propagation characterization using the array response of PN-sequences," IEEE Trans. Wirel. Commun., vol. 15, no. 1, pp. 341-356, 2016.

[19] G. G. Raleigh and T. Boros, "Joint space-time parameter estimation for wireless communication channels," IEEE Trans. Signal Process., vol. 46, no. 5, pp. 1333-1343, 1998.

[20] X. Yin, L. Ouyang, and H. Wang, "Performance comparison of SAGE and MUSIC for channel estimation in direction-scan measurements," IEEE Access, vol. 4, pp. 1163-1174, 2016.

[21] A. Nehorai and E. Paldi, "Vector-sensor array processing for electromagnetic source localization," IEEE Trans. Signal Process., vol. 42, no. 2, pp. 376-398, 1994.

[22] K. T. Wong and M. Zoltowski, "Uni-vector-sensor ESPRIT for multisource azimuth, elevation, and polarization estimation," IEEE Trans. Antennas Propag., vol. 45, no. 10, pp. 1467-1474, 1997.

[23] B. Yang, P. Zhang, H. Wang, and W. Hong, "Efficient delay and AoA estimation using vector antenna for radio propagation measurements," in 2019 IEEE International Symposium on Antennas and Propagation and USNC-URSI Radio Science Meeting, Atlanta, GA, USA, July 2019, pp. 2125-2126.

[24] _ _ "Electromagnetic vector antenna array-based multi-dimensional parameter estimation for radio propagation measurement," IEEE Wirel. Commun. Lett., vol. 8, no. 6, pp. 1608-1611.

[25] G. Zheng, "Two-Dimensional DOA Estimation for Polarization Sensitive Array Consisted of Spatially Spread CrossedDipole," IEEE Sensors Journal, vol. 18, no. 12, pp. 5014-5023, 2018. 
[26] J. Duplouy, C. Morlaas, H. Aubert, P. Potier, and P. Pouliguen, "Wideband vector antenna for dual-polarized and threedimensional direction-finding applications," IEEE Antennas Wirel. Propag. Lett., vol. 18, no. 8, pp. 1572-1575, Aug 2019.

[27] K. T. Wong and X. Yuan, "Vector cross-product direction-finding with an electromagnetic vector-sensor of six orthogonally oriented but spatially noncollocating dipoles loops,” IEEE Trans. Signal Process., vol. 59, no. 1, pp. 160-171, 2011.

[28] P. Stoica and A. Nehorai, "MUSIC, maximum likelihood, and Cramer-Rao bound," IEEE Trans. Acoust. Speech Signal Process., vol. 37, no. 5, pp. 720-741, 1989.

[29] X. Zhang, C. Chen, and J. Li, "Blind DoA and polarization estimation for polarization-sensitive array using dimension reduction MUSIC," Multidimensional Systems and Signal Processing, vol. 25, no. 1, pp. 67-82, 2014. 